Research Article

\title{
Effects of Time-Varying Fluid on Dynamical Characteristics of Cantilever Beams: Numerical Simulations and Experimental Measurements
}

\author{
Mingyu Shao $(\mathbb{D}$, Chicheng Ma $\mathbb{D}$, Shuaizhao Hu $(\mathbb{D}$, Chuansong Sun $(\mathbb{D}$, and Dong Jing \\ School of Transportation and Vehicle Engineering, Shandong University of Technology, Zibo, China \\ Correspondence should be addressed to Chicheng Ma; machch@sdut.edu.cn
}

Received 8 October 2020; Revised 29 November 2020; Accepted 6 December 2020; Published 21 December 2020

Academic Editor: Mehmet Emir Koksal

Copyright (C) 2020 Mingyu Shao et al. This is an open access article distributed under the Creative Commons Attribution License, which permits unrestricted use, distribution, and reproduction in any medium, provided the original work is properly cited.

\begin{abstract}
In order to obtain the effects of time-varying fluid on dynamical characteristics of cantilever beams, this paper gives a comprehensive study of cantilever beams vibrating in a fluid with variable depth. The mathematical model of the cantilever beams in time-varying fluid is derived by combining Euler-Bernoulli beam theory and velocity potential theory, and the influence of the time-varying fluid is discussed. Then, a two-way fluid-structure interaction (FSI) numerical simulation procedure is proposed to calculate the transient responses of the beam. The validity and accuracy are verified according to the comparison among theoretical analysis, numerical simulations, and experimental measurements. Results show that, besides the added mass effect, a damping-like term is also induced due to the motion of the fluid, which is proportional to the moving velocity of the fluid. Both the added mass and the added damping increase with the increment of the width of the beam. The surrounding fluid near the free end affects the beam more significantly. As a negative damping is caused while the fluid decreases, resulting in a much slower decay of the time responses. Therefore, the added damping should not be neglected in the analysis of the FSI problems with timevarying fluid.
\end{abstract}

\section{Introduction}

Fluid-structure interaction is widely encountered in ocean engineering and petroleum engineering, and the fluid induced noise and vibration is a common and difficult problem for engineers. More importantly, in many cases, the surrounding fluid changes or moves fast; therefore, the interaction effect becomes time varying as well. While the submarine dives, the ballast tanks of the submarine are flooded with water. At this moment, the structures of the submarines suffer a time-varying surrounding fluid, and the upper rudder vibrates with increasing fluid. As the submarine surfaces, the rudder and the retractable hydroplanes vibrate with decreasing fluid. The underwater-launched missiles and marine oil pipelines in offshore oil-drilling also work with the variable surrounding fluid, which affects the vibration of the structures significantly. Therefore, it is necessary to study the dynamic characteristics of the structures interacting with the time-varying fluid, and based on that, the vibration of the system can be suppressed.

The influence of fluid impact loads becomes more complex and notable with the increment of the sizes of ships and offshore plants. As a result, many valuable efforts have been devoted to study fluid-structure interaction phenomena theoretically, numerically and experimentally. One of the most important roles of surrounding fluid is the added mass effect, and the added mass sometimes is much larger than the self-weight of structures [1]. Liang et al. [1] presented a simple procedure to estimate the natural frequency and the mode shapes of the submerged cantilever plates based on the empirical formulation. Vu et al. [2] studied the effects of added mass on the submerged plate by experiments, and the influence of the geometry ratios and boundary conditions were also investigated. Kerboua et al. [3] established an analytical model to assess the added mass of rectangular plates coupled with fluid, the finite element 
method was used to model the structure, while the velocity potential theory was adopted to solve the fluid pressure. Rezazadeh et al. [4] modeled the inertial effects of the surrounding fluid on the microbeam as added mass by applying "three-dimensional aerodynamic theory", and the added mass was calculated for various aspect ratios of microbeams. Tariverdilo et al. [5] derived the added mass and frequencies for a clamped circular plate contacting with incompressible fluid and investigated the effect of fluid depth on the coupled system. Based on FourierBessel series expansion and linear potential theory, Shabani et al. [6] proposed an analytical model to investigate the free vibrations of a cantilever microbeam submerged in fluid, demonstrating that the fluid influenced the higher modes more effectively than the lower modes. Golzar et al. [7] studied the dynamic instability of cantilever microbeam submerged in incompressible viscous fluid, and pull-in conditions of the beam were studied. Baroudi et al. [8] developed an analytical model to study the added mass and mode shapes of a fixedfixed beam carrying concentrated masses submerged in fluid, in which the beam was modeled using the Bernoulli-Euler equation, and the fluid was modeled using the acoustic equation. Faria et al. [9] analyzed the energy transport in beam-like structures, and they found that the mode shapes were not significantly affected.

The dynamics of the submerged elastic beams underpin the operation of many modern technology devices ranging from micromechanical sensors to the atomic force microscope (AFM), and the viscosity of the fluid media has a strong influence on those dynamics [10]. Widely relevant applications entail the problems of the coupling motions of slender structures immersed in viscous fluids, and special attention has been focused on the investigations of nano-/ microsystems [11-14]. By simplifying the beam as a string of spheres, Hosaka et al. [15] calculated the damping force due to the surrounding fluid analytically. Compared with the sphere spring, the fluid dynamics of the cylinder was more closed to that of beams with rectangular section. Kirstein et al. [16] presented an investigation on the influence of viscosity on the vibration of microcantilevers with cylindrical cross section, and the added mass and damping were obtained in closed form, which agrees well with the experimental data. Based on the correction of the hydraulic function of the circular beam, Sader [17] presented a detailed study of the frequency characteristics of a cantilever which was submerged in viscous fluid. Chon et al. [18] performed detailed measurements of a series of AFM cantilever beams vibrating in fluid to test the validity and accuracy of Sader's [17] theoretical model; good agreements were found between the experimental data and theoretical results. Considering the general case of a flexible thin blade oscillating in viscous fluid and modeling the fluid motion by linearized NavierStokes equations, Eysden et al. [19] derived the hydrodynamic load for both normal and torsional mode. Aureli et al. [20, 21] conducted a single complex-valued hydrodynamic function to describe the quiescent hydrodynamics loading, and the flexural vibrations of sharp-edge were analyzed with an experimental verification. Phan et al. [22] studied an FSI problem of moderately large oscillation amplitudes, and the added mass and damping effects were modeled through a complex hydrodynamic function. Intartaglia et al. [23] studied the linear vibrations of two identical beams immersed in viscous fluid, and a boundary integral formulation was proposed to compute the hydrodynamic functions. Jalalisendi et al. [24] studied the flow physics due to a thin rigid lamina oscillating in a quiescent viscous fluid, and the particle image velocimetry (PIV) was utilized to investigate added mass and fluid damping phenomena.

In addition to the viscosity effects, the influences of fluid compressibility and solid surface were also investigated widely. Eysden et al. [10] studied the flow fields generating by the motion of flexible cylinders immersed in viscous compressible fluids by theoretical method, and the effects of the fluid compressibility were discussed. Eysden et al. [25] also presented a rigorous theoretical model to predict the frequency response of a cantilever beam oscillating in compressible fluid, and the results showed that fluid compressibility became increasingly important as the mode number rose. Liao et al. [26] studied the vibration characteristics of an elastic container coupling with compressible inviscid fluid, and the influence of fluid compressibility was examined. The fluid is commonly assumed to be unbounded in the theoretical model. However, Green's [27, 28] study showed that a nearby solid surface could significantly affect the frequency response of the cantilever. Naik et al. [29] investigated the dynamic response of a cantilever in air and fluorinert liquids near a solid wall by experimental measurements, and the result showed the resonance frequency decreased with the increasing fluid and decreasing dimensional gap height.

Though the theoretical method could give a parameterized description to the FSI problems, it was usually used for simple structures due to the complex dynamic characteristics caused by the flexible vibration of the structures. Nowadays, with the development of the computation technology, numerical simulations show more and more important roles in structural analyzing and fluid hydrodynamic load solving. Liu et al. [30] investigated the vibration of an elastic cylinder in an axial fluid numerically, combining the arbitrary Lagrange-Euler (ALE) Navier-Stokes equations and dynamic equation of an Euler-Bernoulli beam. Based on finite volume method, Facci et al. [31] carried out a detailed nondimensional parameter study on the hydrodynamic load, including plate aspect ratio, Reynolds number, and relative vibration amplitude. Kramer et al. [32] gave an investigation on the influence of material anisotropy and the added mass on the free vibration response of submerged cantilevered composite plates/beams via combined analytical and numerical modeling.

The dynamic characteristics of a cantilever beam or plate partly submerged in the fluid also interested the scholars. Ergin et al. [33] investigated the natural frequencies and mode shapes of cantilever plates partly contact with the fluid; it is found that the wet mode shapes varied slightly from those in vacuo modes. Sedlar et al. [34] derived the added mass of partially submerged structures, combining the classical concept of modal added mass and local added mass. 
Hossain et al. [35] employed the finite element method to simulate the vibration of a mini cantilever beam partially submerged in a viscous media, and the beam was found to be the most sensitive to respond in resonant frequency and $Q$ factor when the beam was submerged by one-third in viscous media. Hossain et al. [36] also applied finite element analysis (FEA) to characterize the rheological properties of viscous materials by predicting the dynamic response of a cantilever beam partially submerged in air and water. Kwak et al. [37] used the virtual mass method to investigate the free flexural vibration of a cantilever plate partially submerged in fluid, and the influence of the virtual mass was expressed in analytical form in terms of the Mathieu function. Ali et al. [38] presented an analytical solution for the vibration characteristics of a delaminated beam partially submerged in a fluid, and the effect of different parameters on the natural frequencies and mode shapes were examined, including the effect of free surface wave, the fluid density, and the material anisotropy.

It should be noticed that the aforementioned studies mainly focused on the added mass due to the inertial fluid loading, which causes variation in the frequency response of a cantilever beam vibrating in fluid. The influences of the fluid viscosity on the frequency and damping phenomena were usually investigated for the AFM cantilever beams in quiescent fluid. Besides, although the dynamic responses of cantilever beams partially immersed in the fluid had have been researched by different methods, the influences of the time varying characteristics of the surrounding fluid were rarely reported.

In this study, the dynamic characteristics of cantilever beams vibrating in time-varying fluid domain are mainly focused on. To model the surrounding fluid, it is firstly supposed that the surrounding fluid changes linearly, as a submarine dives at a constant speed. The added mass is an approximately linear function with the submerged depth; hence, a linear time-varying mass system is to be investigated. In Section 2, the mathematical model of oscillating cantilever beams in a liquid of variable depth is derived using velocity potential theory and Bernoulli-Euler equation. In Section 3 and Section 4, a two-way FSI numerical simulation procedure is established and verified by the theoretical method and experimental measurements. Then, the dynamic responses of the cantilever beams in time-varying liquid are discussed, and the influence of the liquid descending velocity and beam width on the frequency and damping phenomena are also analyzed in Section 5.

\section{Mathematical Model}

In this study, the dynamic characteristics of beams submerged in a time-varying liquid will be investigated, as shown in Figure 1. For the surrounding liquid, water is representatively chosen here, assumed to be incompressible. Two representative types of cantilever beams, namely, Beam I and Beam II, are considered, and the liquid domain is assumed to be time varying. In the case of Beam I, the free end of the cantilever is submerged in the fluid; while in the case of Beam II, the fixed end of the cantilever is submerged. The total length of cantilever beam is $L$, and the submerged part is represented by the depth of liquid contact with beam, $h(t)$. The distances between the beam and the two sides of the container are denoted by $a_{1}$ and $a_{2}$, and the subscript 1 indicates the side $y>0$. The coordinates $x_{1}$ and $x_{2}$ represent the starting position and ending position of the beam surrounding by liquid, respectively. For Beam I, $x_{2}=L$ and $x_{1}$ is time varying, while for Beam II, $x_{1}=0$ and $x_{2}$ is time varying, and the submerged depth can be expressed as $h(t)=x_{2}-x_{1}$.

Considering that the thickness of the beam is much smaller than its length and width, the Euler-Bernoulli theory can be used. As shown in Figure 1, the systems can be described by two parts: the isolated beam and the submerged beam. The density and the viscosity of the air are small and negligible; hence, only the liquid force is considered. Therefore, the motions of the beams are expressed concisely:

$$
\begin{aligned}
& \mathrm{EI}_{z} \frac{\partial^{4} w(x, t)}{\partial x^{4}}+\rho_{b} A \frac{\partial^{2} w(x, t)}{\partial t^{2}}=0, \quad x<x_{1} \text { or } x>x_{2}, \\
& \mathrm{EI}_{z} \frac{\partial^{4} w(x, t)}{\partial x^{4}}+\rho_{b} A \frac{\partial^{2} w(x, t)}{\partial t^{2}}=F(x, t), \quad x_{1}<x<x_{2},
\end{aligned}
$$

where $\rho b$ is the density of the beam structure, $A=b \delta$ is the area of cross section, $b$ and $\delta$ are the width and thickness of the beam, respectively, symbol $E$ denotes the modulus of elasticity, $I z$ is the moment of inertia, and $F(t)$ is the fluid force acting on the beam. In equation (1), $x<x_{1}$ and $x>x_{2}$ represent the unsubmerged part of Bean I and Beam II, respectively. At the fixed end of the cantilever beam, we have the following conditions:

$$
w=0, \frac{\partial w}{\partial x}=0 .
$$

For the submerged part and unsubmerged part of the beam, the continuity conditions are applied at the location of the free surface of the liquid, i.e., $w^{u}=w^{l}, w_{x}^{u}=w_{x}^{l}$, $w_{x x}^{u}=w_{x x}^{l}$, and $w_{x x x}^{u}=w_{x x x}^{l}$, where the subscript denotes the derivative of coordinate $x$, while the superscript $u$ and $l$ denote the upper part and the low part of the beam, respectively. At the free end, the conditions used for a vibrating beam in vacuum are also applied as the vortex developed at the corner is neglected in this study. Hence, at the free end, the boundary conditions can be expressed as follows:

$$
\mathrm{EI} \frac{\partial^{2} w}{\partial x^{2}}=0, \mathrm{EI} \frac{\partial^{3} w}{\partial x^{3}}=0 .
$$

The influence of fluid viscosity is also not considered here, because the shear stress along the depth direction is very small and is neglected for simplicity. With the assumptions, the submerged part of the beam only sustains the positive pressure of the liquid on both sides, and the distributed load per unit length resulting from the liquid can be expressed as [6]

$$
F(x, t)=b\left(p_{2}(x, t)-p_{1}(x, t)\right), \quad x_{1}<x<x_{2},
$$

where $p(x, t)$ is the transient fluid pressure acting at the submerged beam at coordinate $x$ and the subscript 1 and 2 


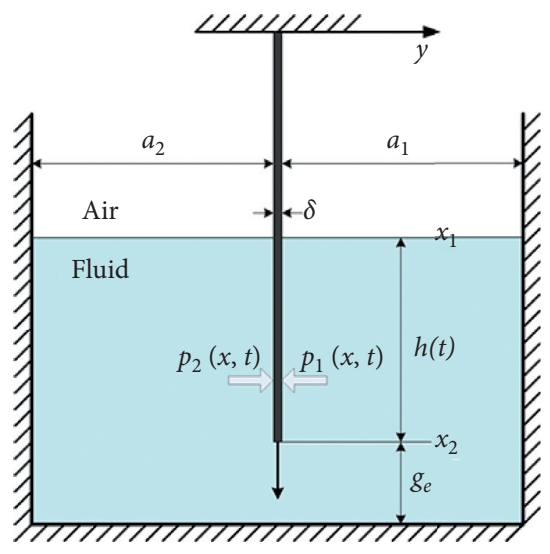

(a)

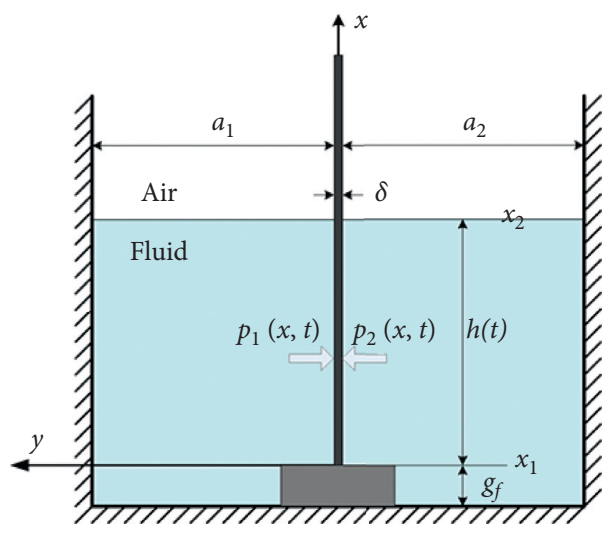

(b)

Figure 1: Cantilever beams in time-varying fluids.

represent two sides of the beam, corresponding to $y>0$ and $y<0$, respectively.

In the liquid domain, the linear potential flow theory is applied to derive the fluid force acting on the beam. The velocity potential function is expressed by the Laplace equation:

$$
\nabla^{2} \phi=\frac{\partial^{2} \phi}{\partial x^{2}}+\frac{\partial^{2} \phi}{\partial y^{2}}+\frac{\partial^{2} \phi}{\partial z^{2}}=0
$$

where $\phi$ is the potential function. Under small deformation conditions, the potential function can be represented by the combination of two functions:

$$
\phi(x, y, z, t)=f(y) g(x, z, t),
$$

From equation (7), it can be found that $f(y)$ is only related to coordinate $y$ and $g(x, z, t)$ is a function of coordinates $(x, z)$ and time $(t)$.

At the contact surfaces between the beam and the surrounding liquid, the velocity of the fluid should match the vibrating velocity of the beam in the transversal direction:

$$
\left.\frac{\partial \phi}{\partial y}\right|_{y=0}=\frac{\partial w}{\partial t}
$$

At the side walls of the container, the no penetration and no slippery conditions are used, and the rigid wall boundary condition is expressed as

$$
\left.\frac{\partial \phi}{\partial y}\right|_{y=a_{1}, a_{2}}=0 .
$$

Solving the Laplace equation (6) and using the boundary conditions (7)-(9), the following expression for the velocity potential function is obtained [3]:

$$
\varphi(x, y, z, t)=\frac{1}{\mu}\left[\frac{e^{\mu(y-2 a)}+e^{-\mu y}}{e^{-2 \mu a}-1}\right] \frac{\partial w}{\partial t},
$$

where $\mu=\pi \sqrt{1 / L^{2}+1 / b^{2}}$. To obtain the pressure function $p$, Bernoulli's equation of the unsteady fluid flow is satisfied at the fluid-structure surfaces [6]:

$$
\frac{\partial \phi}{\partial t}+\frac{1}{2} u^{2}+\frac{p}{\rho_{f}}+g z=0,
$$

where $u$ is the fluid velocity and $z$ denotes the vertical coordinate relative to the reference plane. In the case of small perturbations, the effects of the height change and the nonlinear terms can be neglected; therefore, the fluid positive pressure acting on the structure can be obtained by equation (11):

$$
p=-\left.\rho_{f} \frac{\partial \phi}{\partial t}\right|_{y=0}=-\frac{\rho_{f}}{\mu}\left[\frac{e^{-2 \mu a}+1}{e^{-2 \mu a}-1}\right] \frac{\partial^{2} w}{\partial t^{2}} .
$$

Substituting equation (12) into equation (5), the distributed load per unit length resulting from the surrounding liquid is finally derived as

$$
\begin{aligned}
F(x, t)= & b\left(p_{2}-p_{1}\right)=\frac{\rho_{f} b^{2}}{\pi}\left[\frac{e^{-2 \mu a_{1}}+1}{e^{-2 \mu a_{1}}-1}+\frac{e^{-2 \mu a_{2}}+1}{e^{-2 \mu a_{2}}-1}\right] \\
& \frac{\partial^{2} w(x, t)}{\partial t^{2}} .
\end{aligned}
$$

From the equation (13), one can see that the hydraulic force acting on the beam is proportional to the acceleration, working as an inertia force. This is why we call it added mass effect. Therefore, we can rewrite the equation (13) in the following form:

$$
F(x, t)=-m_{a} \frac{\partial^{2} w(x, t)}{\partial t^{2}},
$$

where $m_{a}$ denotes the additional mass:

$$
m_{a}=-\frac{\rho_{f} b}{\mu}\left[\frac{e^{-2 \mu a_{1}}+1}{e^{-2 \mu a_{1}}-1}+\frac{e^{-2 \mu a_{2}}+1}{e^{-2 \mu a_{2}}-1}\right] .
$$

Equation (15) is derived for a constant fluid. However, if the depth of the liquid changes, the additional mass must be regarded as moving and time varying. It is assumed that the liquid flows in or out of the bottom of the container, and the 
velocity heterogeneity at the bottom of the container has no effect on the whole fluid domain, and then the moving velocity of fluid along the beam can be taken to be a constant value in the entire fluid domain. Considering that the liquid level rises or falls at a constant velocity $v_{0}$ for simplicity (the direction of the liquid motion is positive along the beam coordinate), the moving equation of the beam vibrating in the time-varying fluid domain can be written as $[39,40]$

$$
\mathrm{EI} \frac{\partial^{4} w(x, t)}{\partial x^{4}}+\rho_{b} A \frac{\partial^{2} w(x, t)}{\partial t^{2}}=-\int_{x_{1}}^{x_{2}}\left[m_{a} \frac{\partial^{2} w(x, t)}{\partial t^{2}}+2 m_{a} v_{0} \frac{\partial^{2} w(x, t)}{\partial x \partial t}+m_{a} v_{0}^{2} \frac{\partial^{2} w(x, t)}{\partial x^{2}}\right] \delta\left(x-x^{\prime}\right) \mathrm{d} x^{\prime} \text {. }
$$

The boundary conditions of equation (16) are still the same as those of equations (1) and (2), which are given by equation (3) and (4). The three terms on the right side of the above equation represent the involved inertial force, the Coriolis force, and the centripetal force produced by the moving fluid, respectively. It is found from equation (16) that, besides the added mass effect, the changing liquid plays two actions: one is always positive in the form of $v_{0}^{2}$, denotes additional stiffness; the other is proportional to $v_{0}$, which has the form of additional damping. When the liquid moves along the $+x$ direction, we have $v_{0}>0$; a positive damping-like force is induced, while the velocity of the fluid change is opposite to the $+x$ direction, we have $v_{0}<0$, and a negative damping-like force is induced. In the previous studies involving a constant fluid domain, the coupling equation is much easier. To give an in-depth investigation on the time-varying fluid, here in this study, the entire liquid domain is given as a moving domain, and the influence of the additional force resulting from the motion of the liquid is mainly focused on.

The motion of the cantilever beam can be described using a linear superposition of free vibration modes of the beam:

$$
w(x, t)=\sum_{j=1}^{\infty} q_{j}(t) \varphi_{j}(x),
$$

where $q_{\mathrm{j}}(t)$ is the generalized coordinates and $\varphi_{\mathrm{j}}(x)$ is the mode shape of the $j$-th order of the beam. Shabani et al. [6] proved that using the mode shapes of the cantilever beam in the vacuum supplied an accurate result and here we also use these mode shapes in the vacuum.

Substituting equation (17) into equation (16) and multiplying the equation with $\varphi_{\mathrm{i}}(x)$, the equation of the beam vibrating in the time-varying fluid can be obtained with the integral of $x$ from 0 to $L$ :

$$
\begin{aligned}
& \sum_{j=1}^{\infty} \mathrm{EI}\left[\int_{0}^{L} \varphi_{i}(x) \varphi_{j}^{\mathrm{IV}}(x) \mathrm{d} x\right] q_{j}(t)+\sum_{j=1}^{\infty} \rho_{b} A\left[\int_{0}^{L} \varphi_{i}(x) \varphi_{j}(x) \mathrm{d} x\right] \frac{d^{2} q_{j}(t)}{\mathrm{d} t^{2}} \\
& \quad=-\sum_{j=1}^{\infty} m_{a}\left[\int_{x_{1}}^{x_{2}} \varphi_{i}(x) \varphi_{j}(x) \mathrm{d} x\right] \frac{d^{2} q_{j}(t)}{\mathrm{d} t^{2}}-\sum_{j=1}^{\infty} 2 m_{a} v_{0}\left[\int_{x_{1}}^{x_{2}} \varphi_{i}(x) \varphi_{j}^{\prime}(x) \mathrm{d} x\right] \frac{d q_{j}(t)}{\mathrm{d} t}-\sum_{j=1}^{\infty} m_{a} v_{0}^{2}\left[\int_{x_{1}}^{x_{2}} \varphi_{i}(x) \varphi_{j}^{\prime \prime}(x) \mathrm{d} x\right] q_{j}(t) .
\end{aligned}
$$

The equation (18) can be rewritten in a matrix form as follows:

$$
\left(\mathbf{M}_{\mathbf{0}}+\mathbf{M}_{a}\right) \frac{d^{2} \mathbf{q}(t)}{d t^{2}}+\mathbf{C}_{a} \frac{d \mathbf{q}(t)}{d t}+\left(\mathbf{K}_{\mathbf{0}}+\mathbf{K}_{a}\right) \mathbf{q}(t)=0
$$

where the detail values of each term in the equation are of the following forms:

$$
\begin{aligned}
& M_{0 i j}=\rho_{b} A \int_{0}^{L} \varphi_{i}(x) \varphi_{j}(x) \mathrm{d} x, \\
& K_{0 i j}=\mathrm{EI} \int_{0}^{L} \varphi_{i}(x) \varphi_{j}^{I V}(x) \mathrm{d} x, \\
& M_{a i j}=m_{a} \int_{x_{1}}^{x_{2}} \varphi_{i}(x) \varphi_{j}(x) \mathrm{d} x, \\
& C_{a i j}=2 m_{a} v_{0} \int_{x_{1}}^{x_{2}} \varphi_{i}(x) \varphi_{j}^{\prime}(x) \mathrm{d} x, \\
& K_{a i j}=m_{a} v_{0}^{2} \int_{x_{1}}^{x_{2}} \varphi_{i}(x) \varphi_{j}^{\prime \prime}(x) \mathrm{d} x .
\end{aligned}
$$

In fact, the values of the elements in $K_{a}$ are much smaller than those in $K_{0}$, and its effects on the stiffness of the FSI system can be ignored. The natural frequency of the beam vibrating in the time-varying fluid, and the additional damping due to the motion of the surrounding fluid can be solved by equation (19).

The natural frequency of the beam submerged in the fluid can be solved by the following eigenvalue problem:

$$
\operatorname{Det}\left(\left[\mathbf{K}_{0}-\omega_{f}^{2}\left(\mathbf{M}+\mathbf{M}_{a}\right)\right]\right)=0 \text {. }
$$

It has been found that the natural frequency of the beam vibrating in the fluid and in vacuum are related as follows [1]:

$$
\omega_{f}=\omega_{v} \sqrt{\frac{1}{1+\Gamma_{n}}},
$$

where $\Gamma_{n}$ is the nondimensional additional mass factor which is defined as $\Gamma_{n}=m_{a} / m$. When the length of the beam 
is much greater than its width, parameter $\mu$ can be approximated to $\mu=\pi / b$. Then, for the $1^{\text {st }}$ vibration of the beam, the additional mass factor per unit length is

$$
\Gamma_{n}=-\frac{1}{\pi} \frac{\rho_{f} b}{\rho_{s} \delta}\left[\frac{e^{-2 \pi a_{1} / b}+1}{e^{-2 \pi a_{1} / b}-1}+\frac{e^{-2 \pi a_{2} / b}+1}{e^{-2 \pi a_{2} / b}-1}\right],
$$

Liang et al. [1] gave the additional mass per unit span of the $1^{\text {st }}$ vibration of the cantilever beams:

$$
m_{a}=0.25 \pi \rho_{f} b^{2}, \Gamma_{n}=0.25 \pi \frac{\rho_{f} b}{\rho_{s} \delta} .
$$

\section{Numerical Method and Verification}

Calculating the unsteady load of the surrounding fluid on the cantilever beam by a theoretical method is still a big challenge, especially when the beam is vibrating in the viscous time-varying fluid. Benefiting from the development of numerical methods, it is an effective way to obtain the transient signals from a two-way fluid-structure interaction simulation [41, 42], and then the dynamical parameters can be analyzed based on the numerical results, such as the vibration frequency, added mass, and damping parameters.

3.1. Computational Model. For two different types of cantilever beams vibrating in liquid with variable depth, the computational domains and the boundary conditions of the FSI system are displayed in Figures 2(a) and 2(b), respectively. In Figure 2(a), the Fixed boundary condition is used at the upper end of Beam I, and the other surfaces of the beam that contact with water or air is specified as Fluid-Solid Coupling boundary condition. All the sides of the fluid domain are set to Wall boundary condition, corresponding to the boundary of the container, while the Pressure-Outlet boundary is used to the top of the fluid domain, with a Backflow condition set as to the standard atmosphere. In the present study, the cantilever beams vibrating in liquid with both constant length and variable length are simulated. For the beams submerged in constant liquid, Wall boundary is applied to the bottom of the domain. While the Velocity Inlet is utilized at the bottom to investigate beams immersed in a time-varying liquid, the flow rate of the liquid is specified by Users Defined Functions (UDF). The computational domain and the boundary conditions of Beam II are similar to those of Beam I; however, the lower end of the beam is fixed while the top end is free, which is shown in Figure 2(b).

The fluid domain is discretized with structured grid, and the initial mesh of the fluid domain of different FSI system is given in Figures 3(a) and 3(b), respectively. A relatively coarse mesh is utilized to speed up the calculation, while the domain close to the beam especially the submerged part is meshed finely, and the number of the total elements varies with the initial depth of the liquid.

3.2. Governing Equation of the Fluid Domain. In the two-way fluid-structure interaction simulation, the force of the fluid acting on the solid is obtained by solving the NaiverStokes equations based on the finite volume method. In view of the change of fluid domain, the dynamic mesh is utilized to update the mesh and the boundary conditions. Using the finite volume method, the integral forms of the continuity and momentum equations are written as [41]

$$
\begin{aligned}
& \frac{\partial}{\partial t} \int_{V} \rho_{f} d V+\int_{S} \rho_{f}\left(\vec{u}-\vec{u}_{m}\right) \cdot \vec{n} d S=0, \\
& \frac{\partial}{\partial t} \int_{V} \rho_{f} \vec{u} d V+\int_{S} \rho_{f} \vec{u}\left(\vec{u}-\vec{u}_{m}\right) \cdot \vec{n} d S \\
& =\int_{V} \rho_{f} \vec{f} d V-\int_{S} p \vec{n} d S+\int_{S}(\vec{\tau} \cdot \vec{n}) d S,
\end{aligned}
$$

where $S$ is the boundary of the control volume $V, \vec{u}$ is the velocity vector of the fluid, $\vec{u}_{m}$ is the mesh velocity vector of the moving mesh, $\vec{n}$ is the normal vector to $\mathrm{d} S, p$ is the pressure, and $\vec{\tau}$ is the viscous stress vector.

Different from the previous studies, the free surface in this study is time varying resulting from the descending of liquid and the vibrating of the beam, while the free surface resulting from the sloshing of liquid was widely investigated in the dynamic analysis of the ocean waves. Boussinesq-type wave models with improved nonlinearity and dispersion characteristics are effective to simulate the propagation of the waves [43], while some three-dimensional flow characteristics cannot be represented adequately [44]. An alternative way is to solve the Navier-Stokes equations directly with proper free surface tracking techniques [43], such as volume of fluid (VOF) model [45]. The main drawback of the aforementioned method is that it is computationally expensive, although a quite well wave dynamic can be resolved and combined with the proper turbulence model [46]. A direct simplification of the aforementioned approach is to transform the vertical Cartesian coordinate into a vertical coordinate that moves with the free surface, which approximately has the same accuracy as the VOF model in wave height prediction [43]. While another approach is adopting an integral contravariant form of the NaverStokes equations in a generalized curvilinear coordinate system [44].

In this study, the incompressible NavierStokes equations are solved to calculate the hydrodynamic load acting on the structure, and the VOF model is utilized in free surface tracking. The VOF model is a surface tracking technique applied to a fixed Eulerian mesh, which is accomplished by the solution of a continuity equation for the volume fraction of one of the phases. For the $n$-th phase, the continuity equation can be express as

$$
\frac{1}{\rho_{n}}\left[\frac{\partial}{\partial t}\left(\alpha_{n} \rho_{n}\right)+\nabla \cdot\left(\alpha_{n} \rho_{n} \vec{u}_{n}\right)=S_{\alpha_{n}}+\sum_{m=1}^{N}\left(\dot{m}_{m n}-\dot{m}_{n m}\right)\right]
$$

where $\dot{m}_{m n}$ is the mass transfer from phase $m$ to phase $n$ and $\alpha_{n}$ is the volume fraction of the $n$-th phase in the cell, which sums to be unity of all phases.

There are different approaches to the VOF model, and some approaches involve full geometrical reconstruction of the interface, while the other approaches are proposed as 


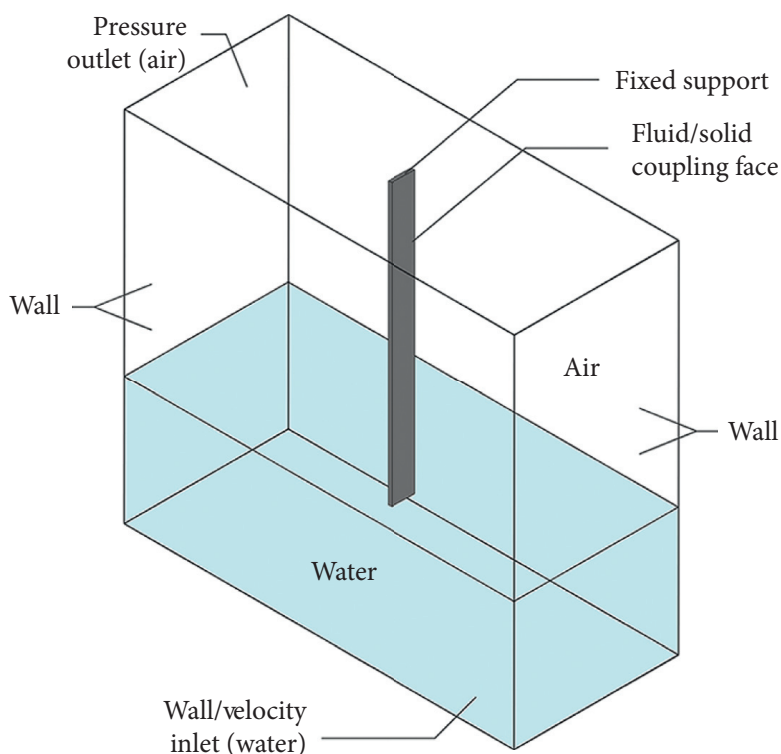

(a)

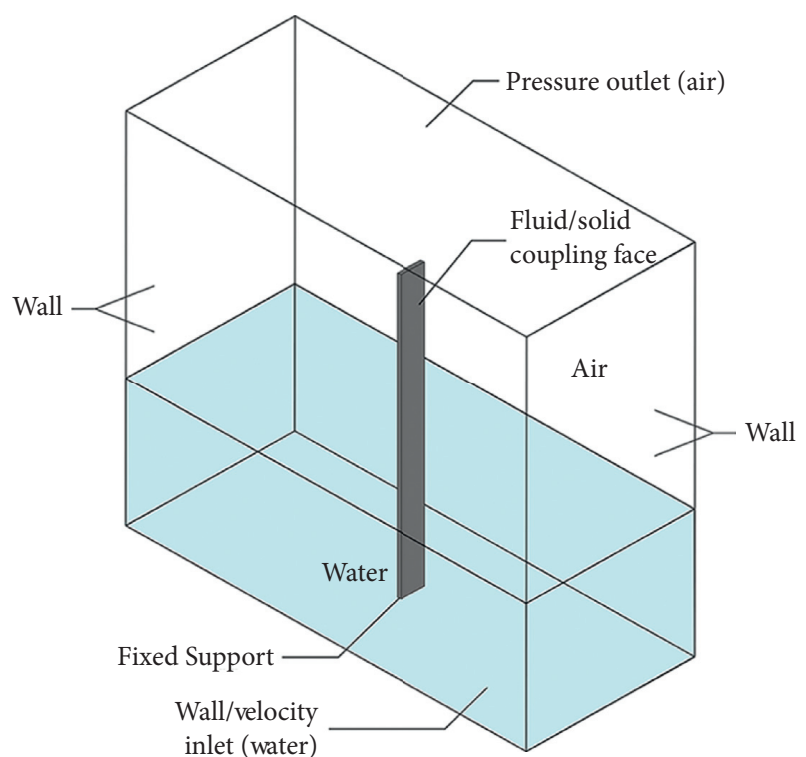

(b)

FIgure 2: Boundary conditions of the FSI system. (a) Beam I; (b) Beam II.

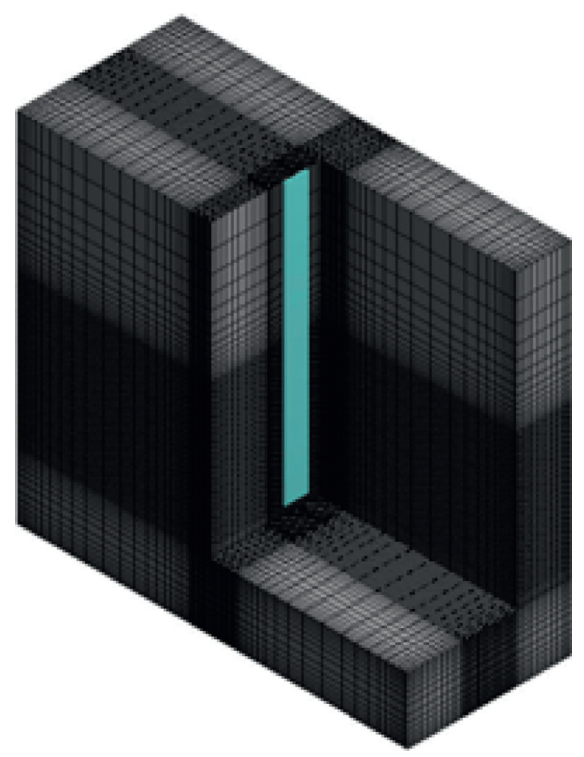

(a)

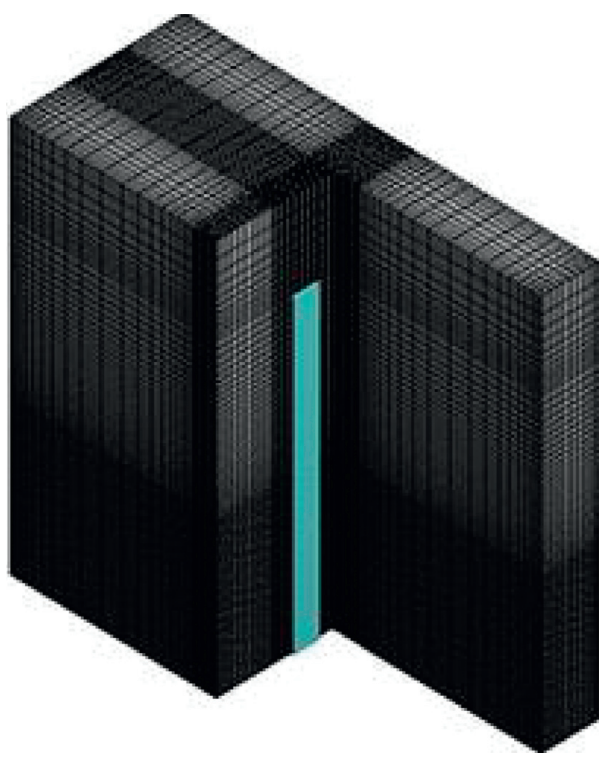

(b)

FIgUre 3: Initial mesh grid of the fluid domain. (a) Beam I; (b) Beam II.

simplified versions of the full VOF model [47]. Assessment of the approaches showed a reasonable dependence between sophistication and accurateness [47]. To ensure the accuracy of the simulation, the Geometric Reconstruction Scheme is adopted in this study. This scheme represents the interface with a piecewise linear approach by assuming that there is a linear slope within each cell between two phases. The Geometric Reconstruction Scheme is the most accurate in Fluent and is applicable for general unstructured meshes [48], while the drawback of this scheme is computational, especially in the FSI simulation due to the iteration between two solvers.

3.3. FSI Method. ANSYS Workbench is a powerful tool to analyze FSI problems, and the joint simulation between the Fluent and Mechanical module is easy to be implemented. We develop the three-dimensional model of a vibrating cantilever beam immersed in a time-varying fluid, based on the two-way fluid-structure coupling calculation. In the calculation, three layers of iterations are included: time loop, 
coupling loop, and field loop. The field loops of fluid and solid are carried out by Fluent and Mechanical module, respectively. The flow chart of the simulation is presented in Figure 4. At the very start, the flow field is initialized and the initial conditions for the solid domain are given. In the $n$-th time step, a UDF function is compiled to update the descending velocity of liquid; meanwhile, the boundary of flow field is replaced according to the displacement of structures. The dynamic mesh method is applied to update the fluid domain mesh. After the iterative calculation of the flow field, the pressure of the flow field is transferred to structural analysis, and then the transient analysis of structures is executed.

For this FSI problem, one of the key difficulties is the data synchronization between fluid solver and solid solver, which is realized by the System Coupling module. This module also controls the iterations of time loop and coupling loop. The convergence is judged by the system coupling module. If it does not converge, the fluid solver is returned to continue the FSI iteration. The calculation iterates continuously until the convergence condition is satisfied or the maximum iteration step is achieved.

The theoretical analysis and numerical results are to be validated through an experimental test, and the responses of the cantilever beams in a constant fluid domain are measured, including the case for Beam I and Beam II. Figure 5 shows the schematic diagram of the platform for vibration measurements of the submerged beams. A cantilever beam is immersed in a tank of acrylic glass for both abovementioned cases. The accelerometer IEPE BZ1141 is installed at L/4 near the fixed end of the beam, and the response of the same point is calculated in the numerical simulation. To obtain a good signal, the signal conditioner BZ 2703 is linked between the accelerometer and the data collector NI-6434. Labview is utilized to visualize and process the vibration signal.

The two beams have the same dimensions. The thickness $\delta$ is equal to $4 \mathrm{~mm}$, and the length $L$ and the width $b$ are $360 \mathrm{~mm}$ and $30 \mathrm{~mm}$, respectively. The length and width of the tank are $500 \mathrm{~mm}$ and $300 \mathrm{~mm}$, and the depth of which is $450 \mathrm{~mm}$, respectively. As shown in Figure 1, the cantilever beams are located at the middle of the horizontal plane of the container, i.e., $a_{1}=a_{2}$. The gap between the free end of Beam I and the bottom of container is $10 \mathrm{~cm}$, while the gap between the fixed end of beam II and the bottom of the container is $4 \mathrm{~cm}$, which is the height of the clamp. The cantilever beam is made of aluminum. The elastic modulus and the density are $68 \mathrm{GPa}$ and $2700 \mathrm{~kg} / \mathrm{m}^{3}$, respectively.

The free vibration response of the cantilever beams submerged in a constant liquid with different depths is measured. An initial displacement is imposed on the beam near the free end for both Beam I and Beam II. When the FSI system stabilizes, the constraint is removed suddenly and the test is triggered. The acceleration signals obtained from the accelerometer are tuned by the signal conditioner and then inputted to Labview by the data collector. The transient responses and the frequency responses can be directly obtained from Labview.

In the two-way FSI simulation, the calculation conditions are basically the same as those in experimental test, while the fixtures of both the two beams are neglected for simplification, and the gap between the bottom of the container and the fixed end of Beam II is approximated to be zero. The transient responses of the cantilever beams immersed in constant fluid and time-varying fluid are simulated. In both cases, the initial deformation of the beam is produced by applying a quasi-static load at the free end slowly. Then, the external load is removed instantaneously, resulting in the vibration of the beam coupling with the constant liquid or time-varying liquid, and the acceleration and displacement signals are recorded.

For the case of Beam I which is vibrating in liquid with a constant submerged depth of $18 \mathrm{~cm}$, Figure 6 shows the pressure contours of the plane which locates at $3 \mathrm{~cm}$ below the free liquid surface. The pressure contours in one period are displayed with the velocities and the accelerations of the beam, demonstrating that the pressure distribution on the two sides is always approximately symmetrical. Hence, it is reasonable to separate the fluid into two independent and identical parts in the theoretical analysis. In Figure 6, one can also see that the action of the surrounding fluid mainly concentrates in a small domain close to the beam. While at the region far away from the beam, it can be assumed the fluid is insignificantly influenced by the vibration of the beam, and the influence of the fluid can be neglected for simplification.

\section{Result of Constant Fluid Domain}

4.1. Frequency Analysis. The $1^{\text {st }}$ natural frequencies of the cantilever beam in constant fluid domain with different submerged depths are studied in Figure 7. The results obtained by different methods are compared based on theoretical method, FSI simulation, and experiment. The results calculated by the method in [1] are also presented. The comparison of the four curves shows that the obtained results agree well with each other. Through the data analysis for beam I, the maximum relative errors of theoretical results and simulation results are $7.66 \%$ and $3.13 \%$ compared with that of tests, while for beam II, the maximum relative errors are $1.19 \%$ and $2.65 \%$, respectively.

It is obvious that with a larger submerged length, the frequencies become smaller resulting from larger added mass effects. But, it is out of our expectation the changes of the frequency are of great difference: while the free end is submerged in the fluid, the frequency decreases fast at $h<L$ / 2 , and the increment of added mass plays an insignificant role if the submerged length exceeds half of the total length of the beam; while the fixed end is submerged in the fluid, the frequency decreases very slowly at $h<L / 2$, and the increment of the added mass plays a larger role if the submerged length exceeds half of the total length of the beam. It can be concluded that the surrounding liquid near the free end of the beam affects the natural frequency characteristics of the FSI system more remarkably, while the liquid surrounding the fixed end of the beam has very little effect on the frequency response of the FSI system.

It can be found that the maximum error of the theoretical results occurs when the submerged depth is small for 


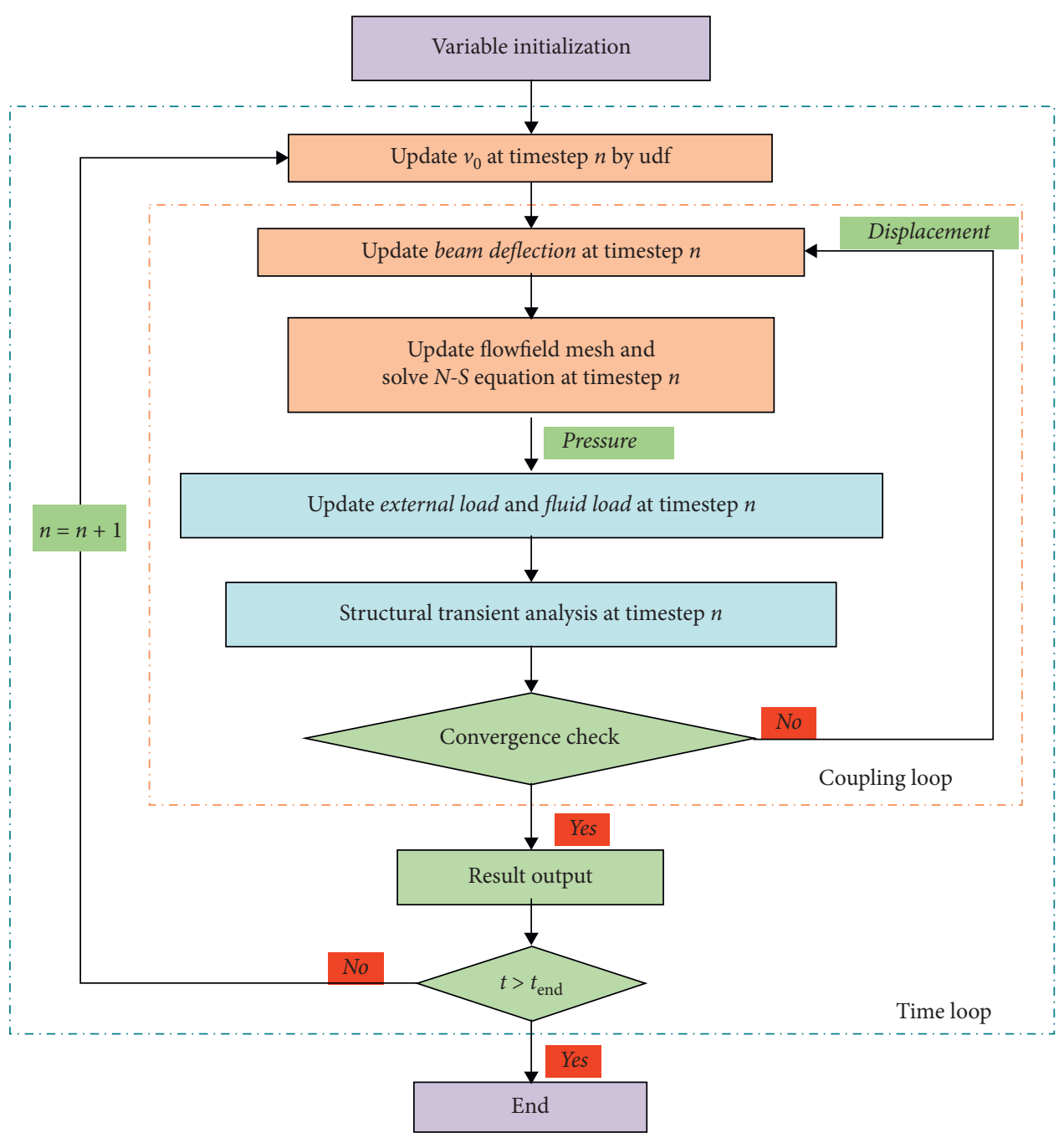

FIgUre 4: Flow chart for fluid-structure interaction calculation.

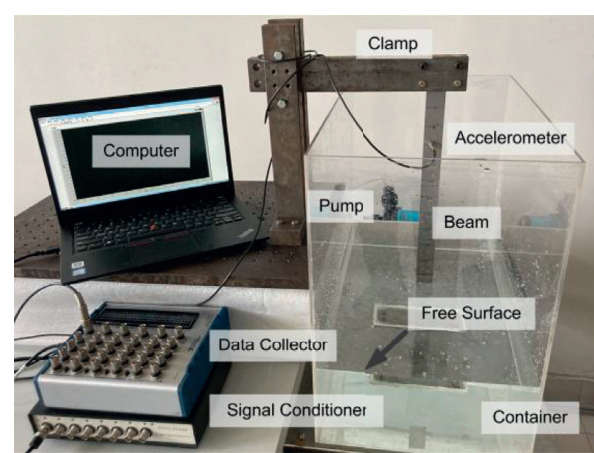

(a)

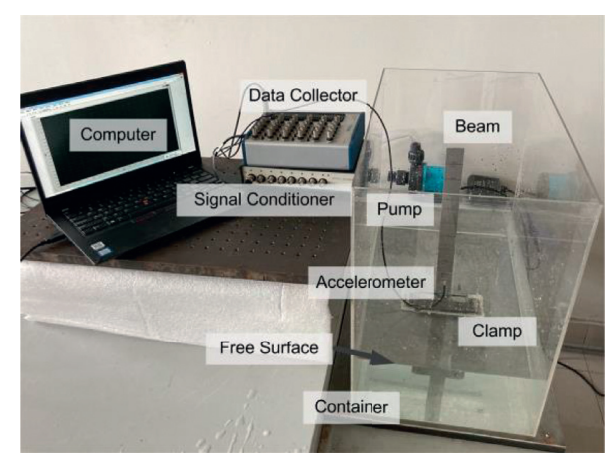

(b)

Figure 5: Experimental measurements. (a) Beam I; (b) Beam II.

Beam I. The error of frequency results from the calculation of the added mass. This is mainly due to the hypothesis that mode shape of the beam in fluid is the same as that in vacuum. Figure $8(\mathrm{a})$ shows the $1^{\text {st }}$ mode shapes of Beam I calculated by theoretical method and FSI simulation. The results obtained by the FSI simulation show that the wet mode shape is slightly different from the dry mode shape of the cantilever beam submerged near the free end. Another possible reason is that the change of the free surface near the beam which is not considered in the theoretical model. It is more significant when the free end is submerged and the submerged depth is small, as shown in Figure 8(b). This 


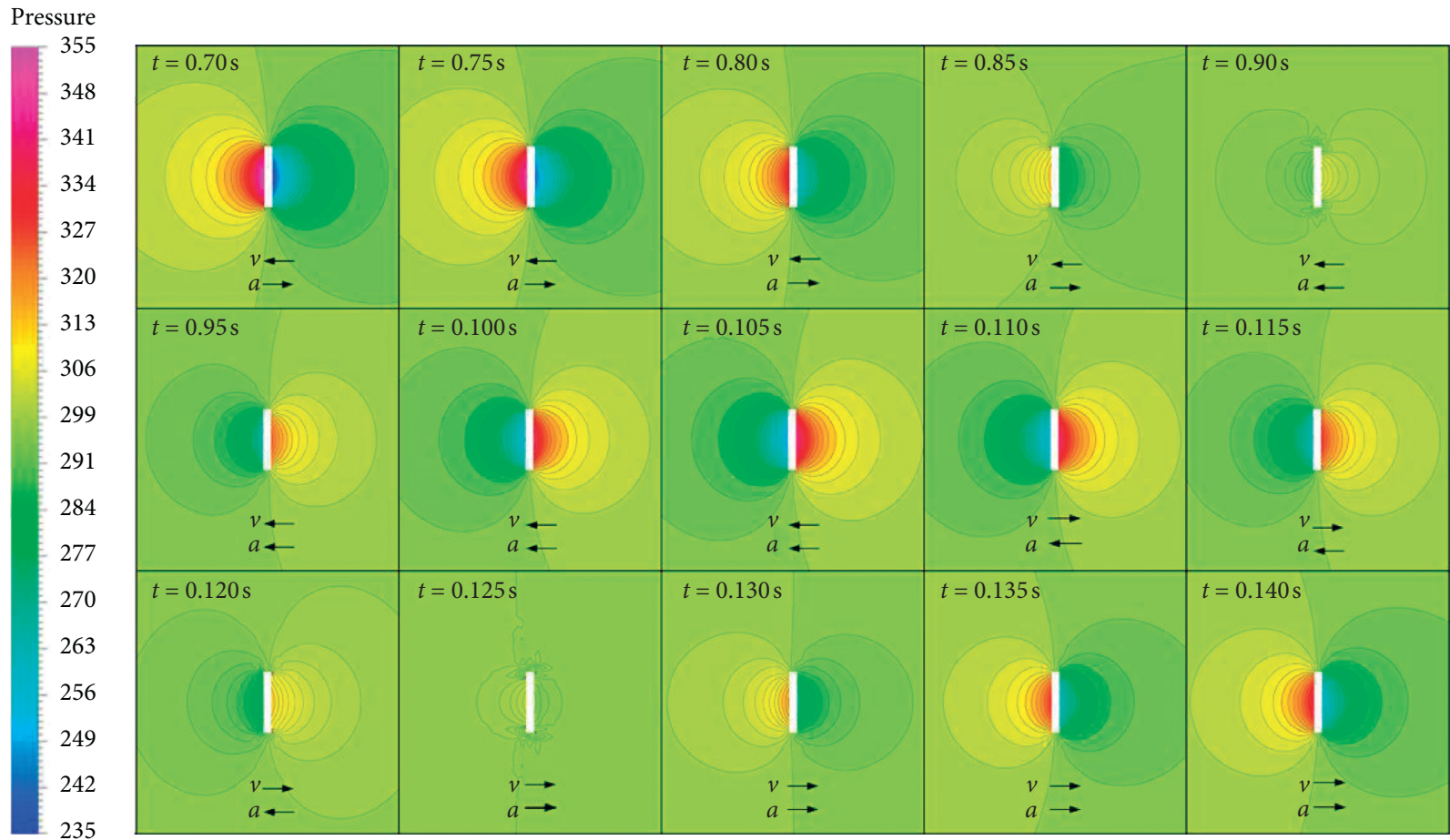

$[\mathrm{Pa}]$

Figure 6: The pressure contour of the FSI system of Beam I $(3 \mathrm{~cm}$ below the free surface, $h=18 \mathrm{~cm})$.

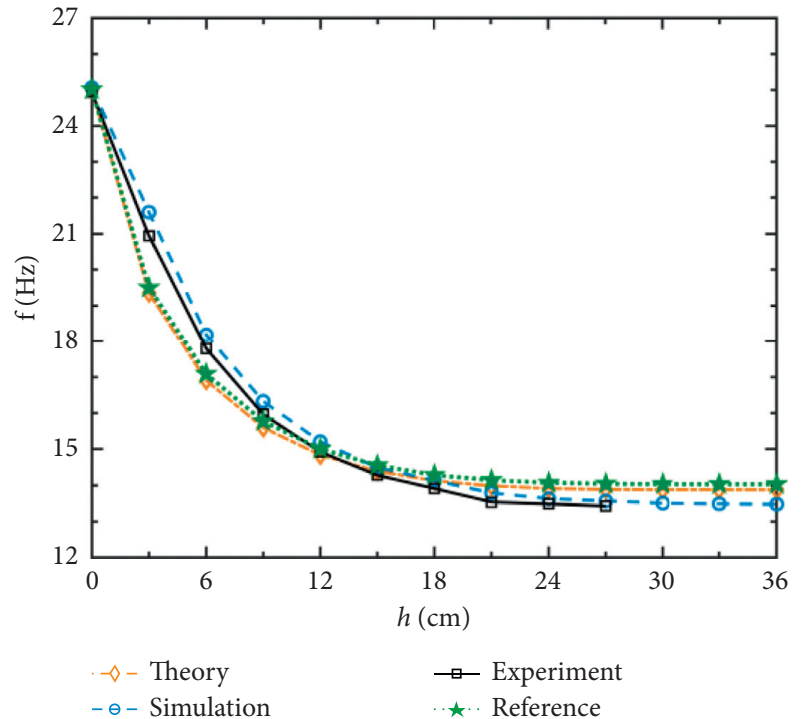

(a)

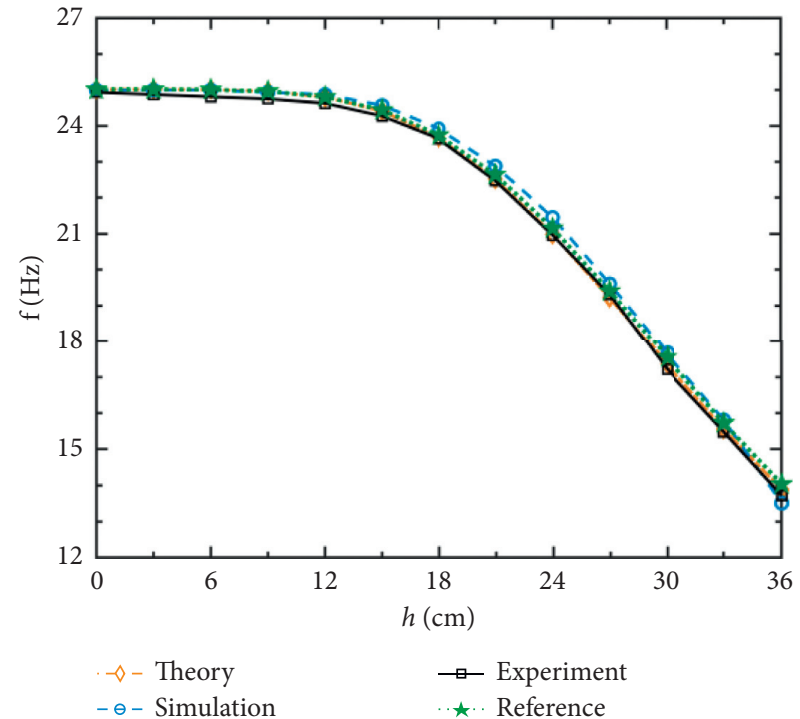

(b)

Figure 7: Comparison of natural frequency of $1^{\text {st }}$ order (different methods). (a) Beam I (b) Beam II.

change may cause a difference of the added mass and result in the error of frequency.

For submerged beams, the influence of the width of the beam is much larger than that of the thickness, as the added mass is proportional to the square of the width (equation (24)). The frequencies of the cantilever beam with different widths are studied by theoretical method and numerical simulation, as shown in Figure 9. The results obtained by theory and simulation are well consistent. When the submerged depth is the same, the vibration frequencies decrease with the increment of the width of the beam. The explanation is that the additional mass factor increases with the width of the beam, as shown in equations (23) and (24), causing the decrement of the vibration frequencies. 


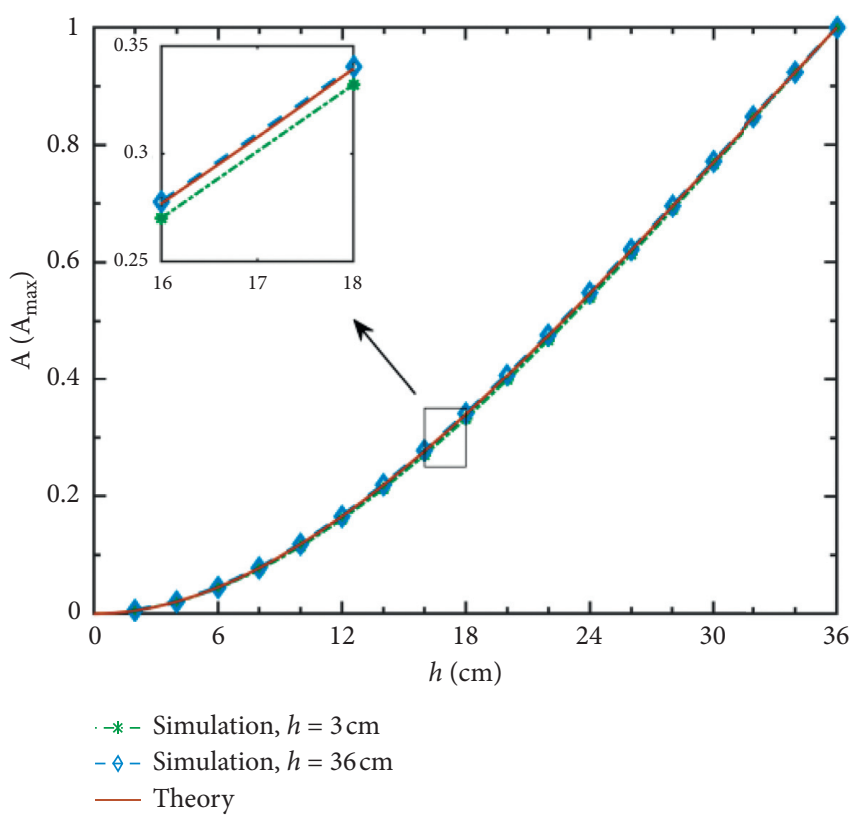

(a)

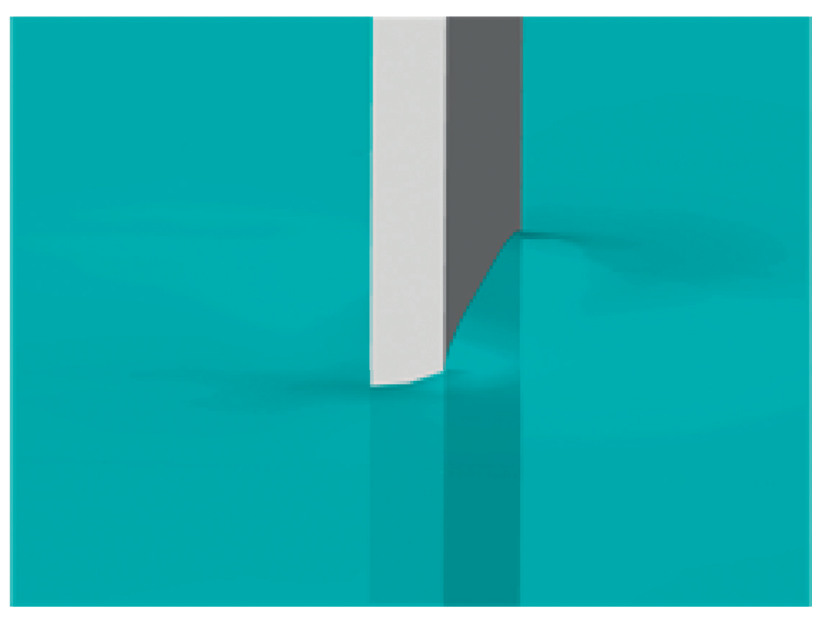

(b)

Figure 8: (a) 1st mode shape of Beam I calculated by different methods; (b) free surface near the beam (Beam I: $h=18 \mathrm{~cm}, t=0.125 \mathrm{~s}$ ).

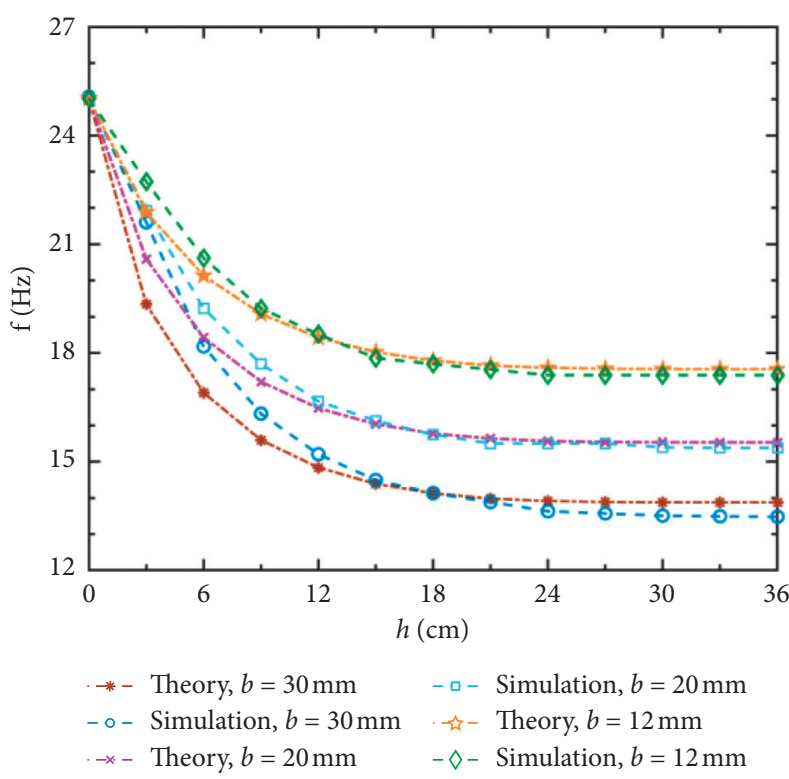

(a)

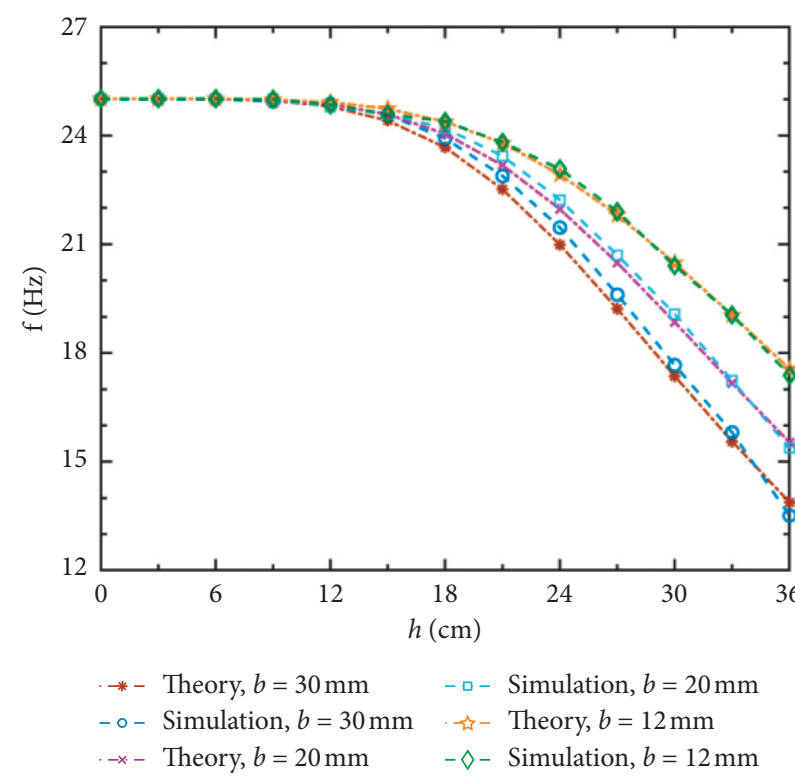

(b)

FIGURE 9: Comparison of natural frequency of 1st order (different widths). (a) Beam I (b) Beam II.

4.2. Transient Analysis. In this section, transient analysis of a beam vibrating in a constant fluid domain is given. For Beam I and Beam II, a constant submerged depth of $18 \mathrm{~cm}$ is applied. The acceleration is measured in the experiment, and the normalized acceleration is plotted. The normal amplitude of the acceleration is also obtained using nonlinear least squares fitting. Comparison of numerical simulation and the experimental measurements are displayed in Figure 10. It is found that the numerical results show good agreement with the experimental data. The damping ratios are also calculated from the logarithmic decrement of the vibration response. For beam I, the relative error of the damping ratio calculated by the simulation method is $7.43 \%$, which is $13.04 \%$ for beam II. The errors mainly come from two sources: the interference in the experiment and the approximate value of the viscosity in the simulation. One can see that the damping ratio of Beam II is much smaller than that of Beam I, which implies that the damping 


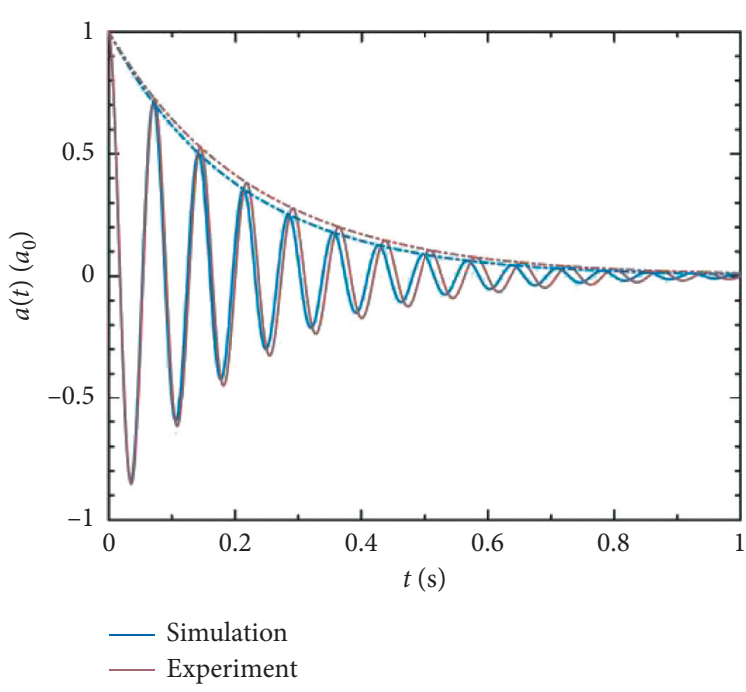

(a)

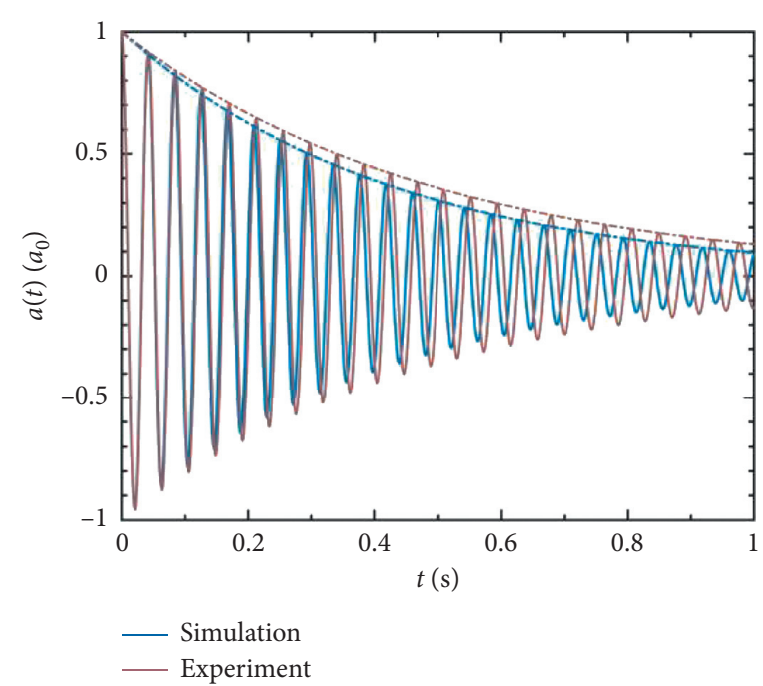

(b)

Figure 10: Time history of normalized accelerations by simulation and experimental measurement. (a) Beam I; (b) Beam II.

characteristics of the submerged cantilever beams are also mainly affected by the viscous effects of the surrounding liquid near the free end. Therefore, the simplification in the FSI simulation that $g_{f}=0$ for the case of Beam II is reasonable.

The comparison demonstrates that the trends of hydrodynamic pressure and the viscous stress from the simulation agree well with the experimental data. The advantage of the FSI simulation is that it can be used in the conditions that are difficult to achieve in the experiment. Further, the dynamic response of both the beam and the fluid can be obtained simultaneously. While a main drawback of the method is computationally expensive. Each curve plotted in Figure 10 was calculated using about 100 hours by our personal computer, as a fine mesh grid is needed in the submerged part of the beam. Based on the verification, the applicability of the present solver can be implemented for the FSI problems involving a time-varying fluid.

\section{Result of Time-Varying Fluid Domain}

The transient responses of the cantilever beams vibrating in the time-varying domain are studied by two-way FSI simulation based on the introduced method. In the simulation for Beam I, an initial submerged depth of $18 \mathrm{~cm}$ is used; while for Beam II, an initial submerged depth of $32 \mathrm{~cm}$ is given, considering that the surrounding fluid influences the two beams in different ways as introduced above. As for the experiment, the obtained signals are noisy because it is difficult to control the flow velocity and the start time correctly. Therefore, in the following studies, only the situation that water flowing out of the container is considered here.

5.1. Results of Different Fluid Velocities. The influences of the fluid moving velocity on the transient characteristics of the FSI system are firstly discussed, and different descending velocities are applied in the simulation, including $0.08 \mathrm{~m} / \mathrm{s}$, $0.16 \mathrm{~m} / \mathrm{s}$, and $0.24 \mathrm{~m} / \mathrm{s}$. The time histories of normalized displacements and amplitudes are plotted in Figures 11 and 12, representing the cases of Beam I and Beam II, respectively. From the frequency analysis, it has been known that the two cases are influenced by the surrounding fluid in different ways. The transient analysis shows the difference as well, consistent with the conclusion obtained from Figure 7.

The descending velocity of the liquid has different effects on the response of two different types of beams, which are mainly reflected in the damping characteristics. A faster decay appears when the fluid flows out of the container quickly for Beam I, while a slower decay appears with a larger descending velocity of fluid for Beam II. The decay of the response is dominated by the inherent damping of the fluid and the added damping caused by the descending of the liquid. The inherent damping decreases with the depth, while the added damping is proportional to $v_{0}$. For Beam I, the vibration decays faster with increment of $v_{0}$ due to the positive added damping induction, which plays a significant role in the transient response. While for Beam II, the vibration decays slower with a larger $v_{0}$, due to the negative added damping. The additional damping-like force cannot be neglected in the dynamic analysis of FSI problems with time-varying fluid. The newly reported phenomenon is important as the modern marine equipment and building structure are greatly influenced by the changing surroundings, and the additional damping-like force plays a much larger role than imagined.

5.2. The Influences of Beam Width. Computed by the proposed simulation technology, the beams of different widths are considered, and the transient responses are solved. The time histories of the normalized displacement and amplitudes are shown in Figures 13 and 14. In the simulation, three different widths of the beam are used, including $0.30 \mathrm{~m}, 0.20 \mathrm{~m}$, and $0.12 \mathrm{~m}$, and the moving velocity of the 

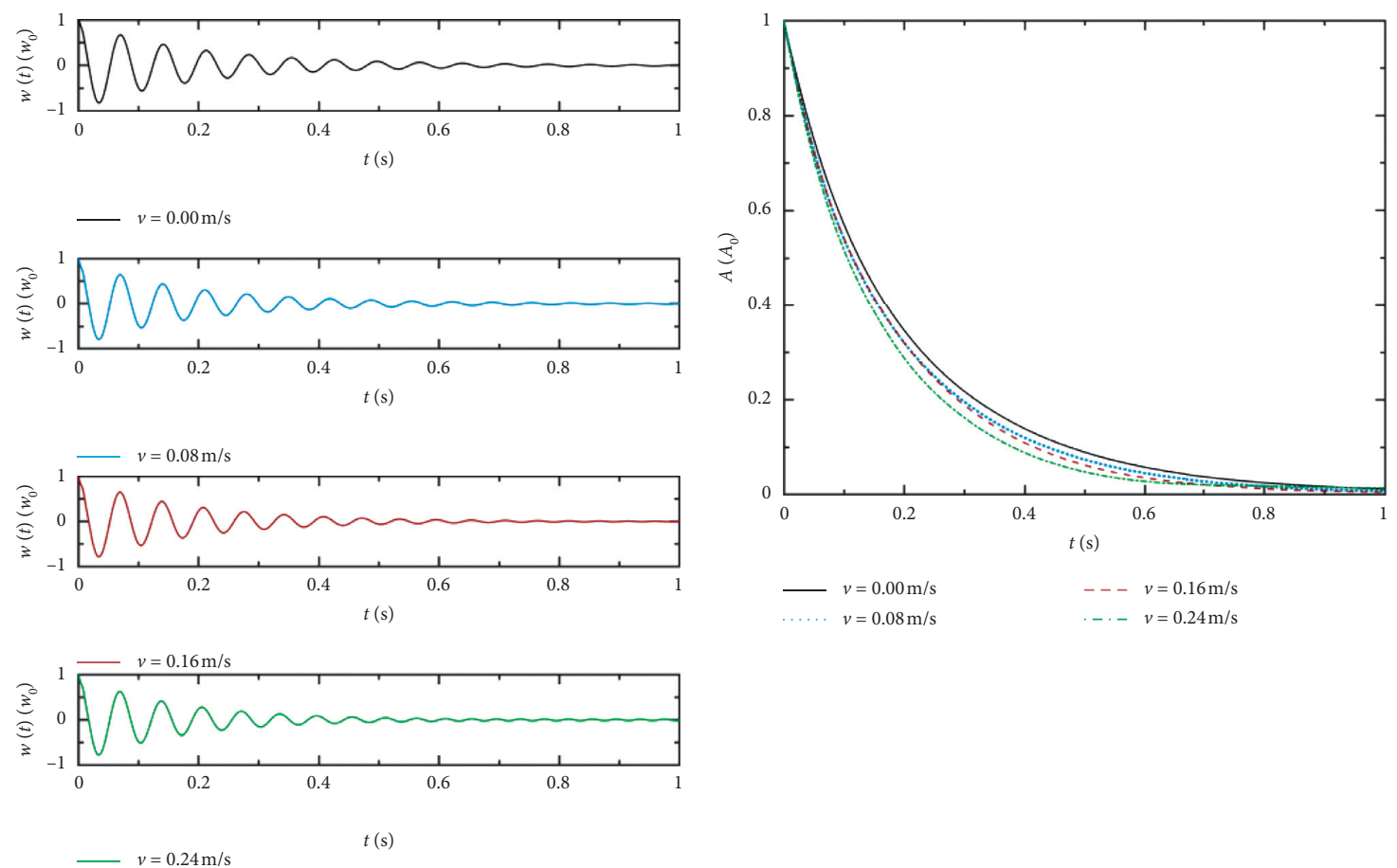

(a)

(b)

FIGURE 11: Transient responses of Beam I with different descending velocities of fluid. Time history of (a) normalized displacements and (b) normalized amplitudes.
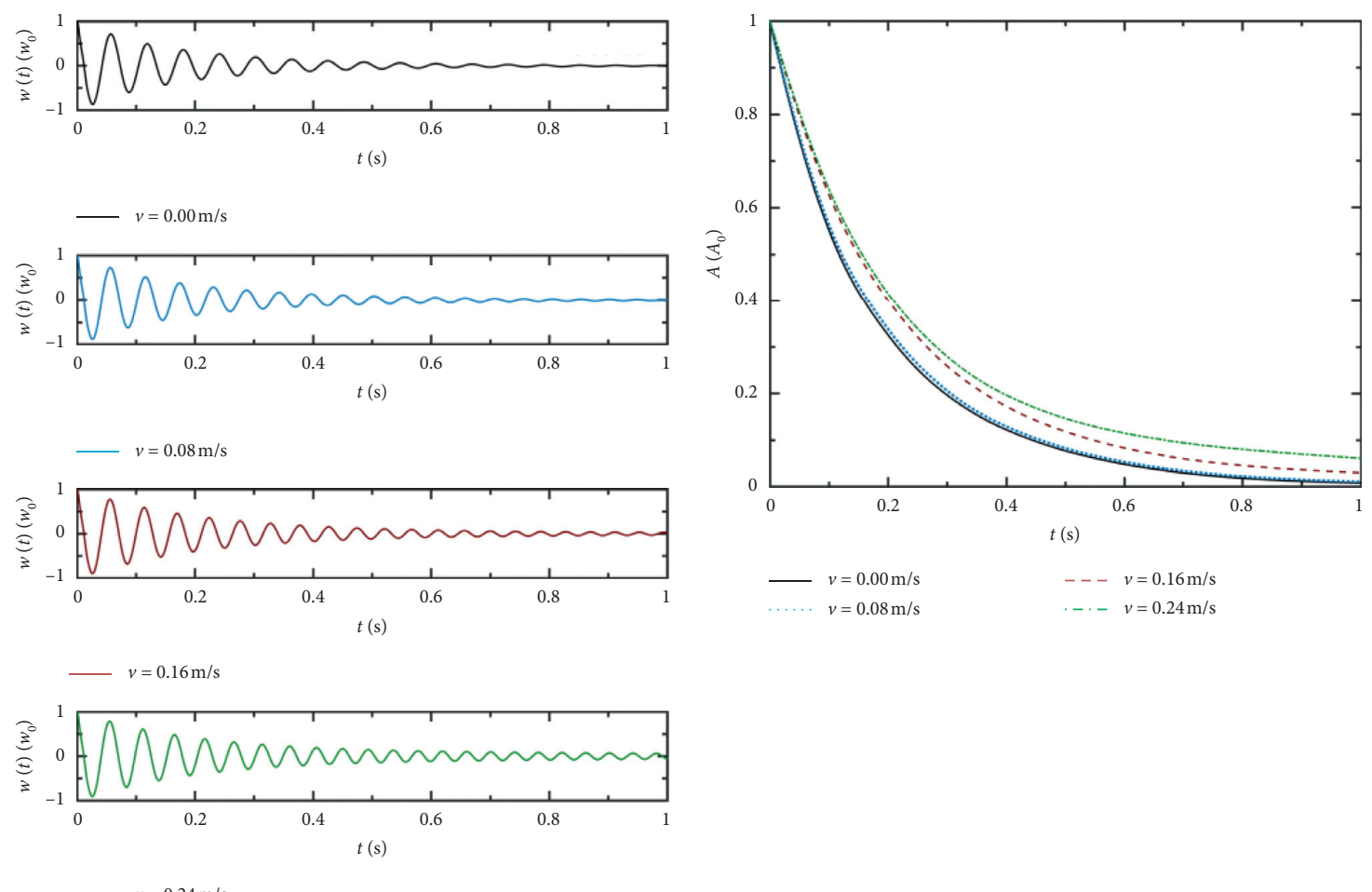

(a)

(b)

FiguRE 12: Transient responses of Beam II with different descending velocities of fluid. Time history of (a) normalized displacements and (b) normalized amplitudes. 

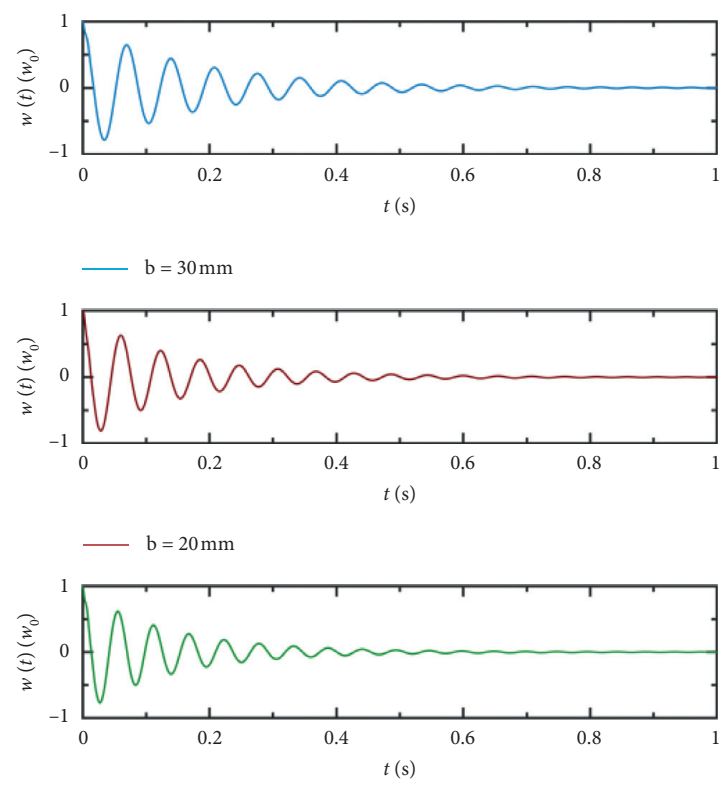

$\mathrm{b}=12 \mathrm{~mm}$

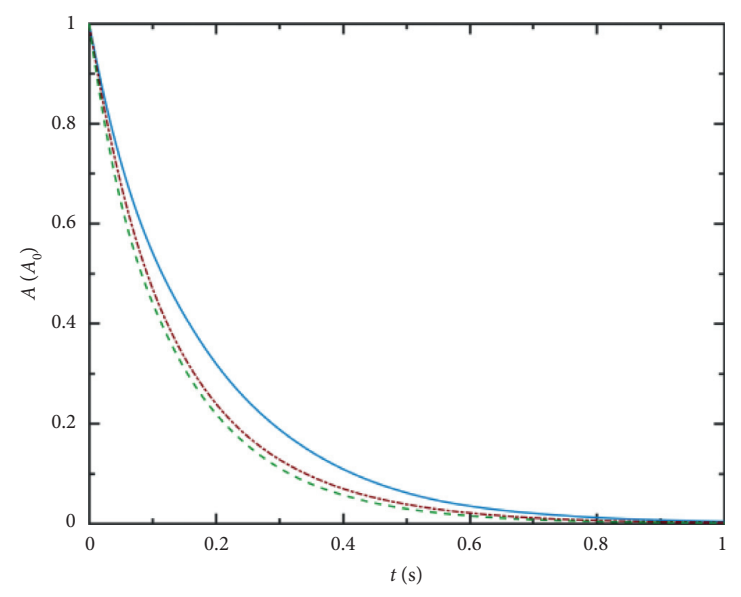

$b=30 \mathrm{~mm}$

-.. $b=20 \mathrm{~mm}$

$-\ldots b=12 \mathrm{~mm}$

(a)

(b)

Figure 13: Transient responses of Beam I with different widths. Time history of (a) normalized displacements and (b) normalized amplitudes.
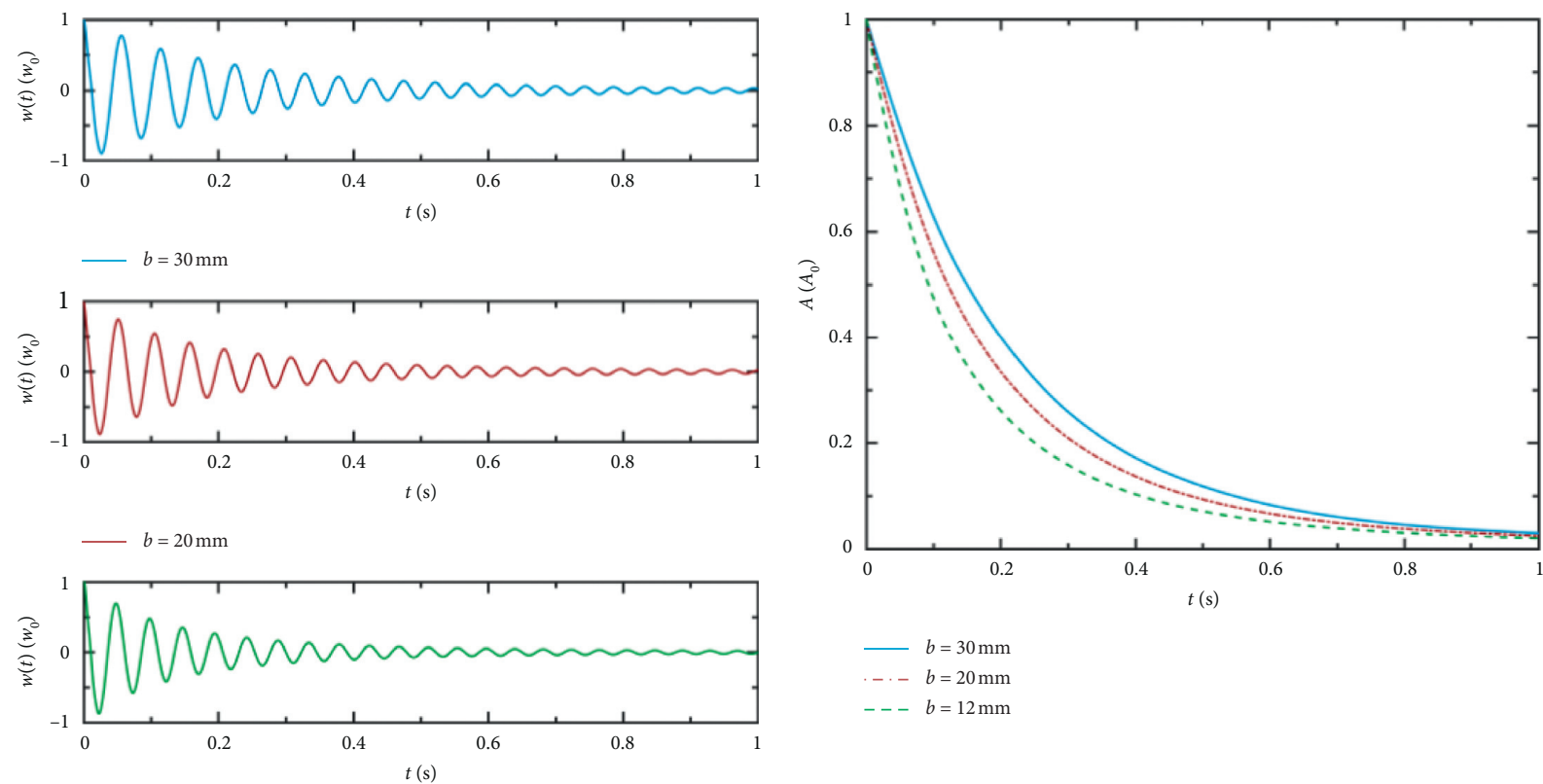

$b=12 \mathrm{~mm}$

(a)

(b)

Figure 14: Transient responses of Beam II with different widths. Time history of (a) normalized displacements and (b) normalized amplitudes.

fluid for both Beam I and Beam II is a constant $v_{0}=0.16 \mathrm{~m} / \mathrm{s}$. Actually, the damping contains two components, the added damping caused by the motion of the fluid and the inherent damping caused by the fluid viscosity. For Beam I in
Figure 13, as the beam has a larger width, the added damping caused by the motion of the fluid increases, which is shown in equations (15) and (20). While the inherent fluid damping decreases with the increment of the kinetic Reynolds 

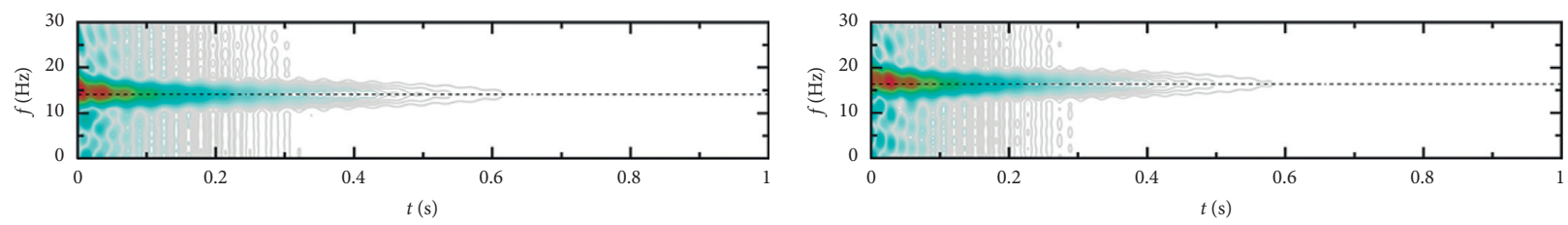

- $v=0.00 \mathrm{~m} / \mathrm{s}$

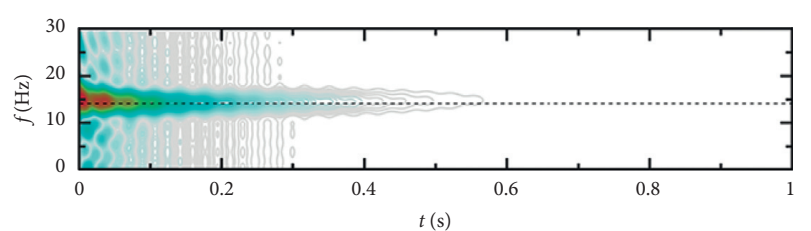

o $v=0.08 \mathrm{~m} / \mathrm{s}$

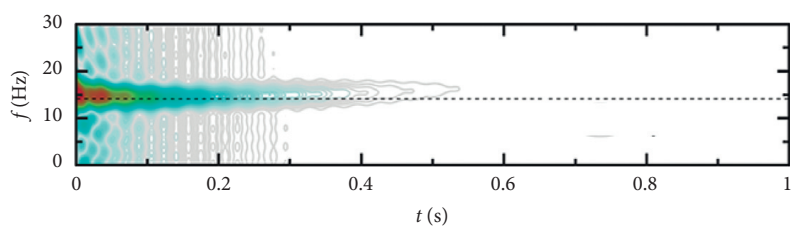

o) $v=0.16 \mathrm{~m} / \mathrm{s}$

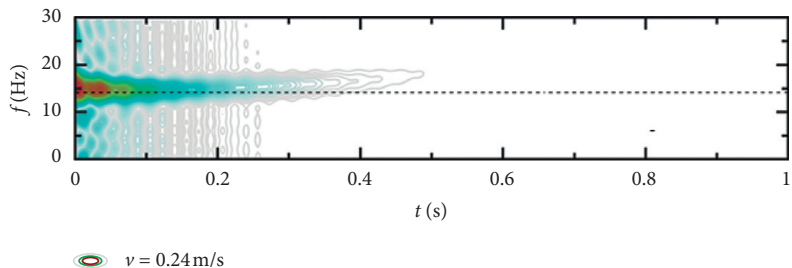

(a)

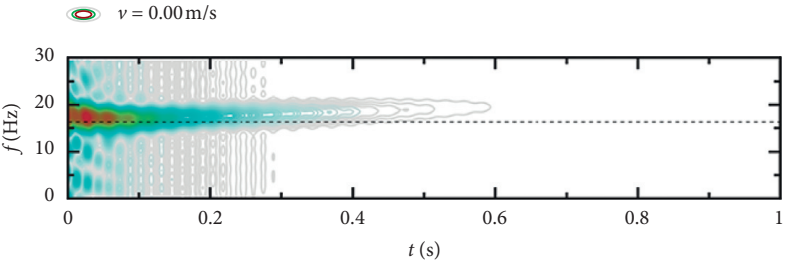

o) $v=0.08 \mathrm{~m} / \mathrm{s}$

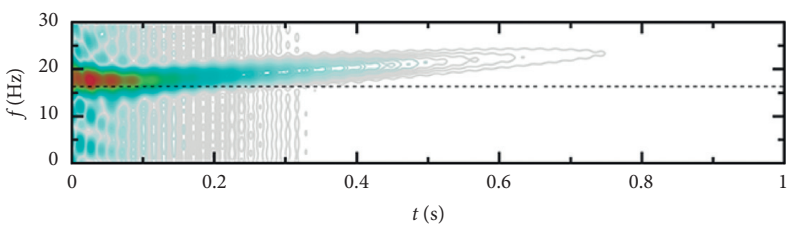

- $v=0.16 \mathrm{~m} / \mathrm{s}$

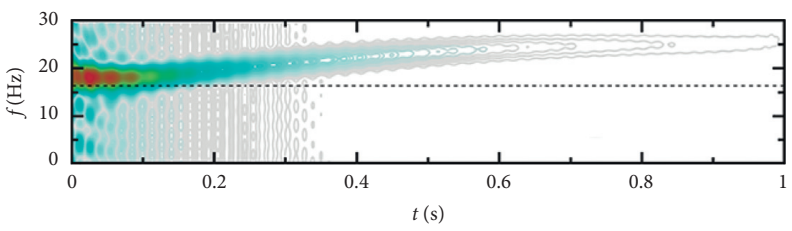

o. $v=0.24 \mathrm{~m} / \mathrm{s}$

(b)

FIGURE 15: Time-frequency analysis of the vibration signals with different descending velocities. (a) Beam I; (b) Beam II.

number which is proportional to the width of the beam [16]. These two caused damping to have opposite effects on the vibration of the beam, which play a complicated role in the transient response of the FSI system. For Beam II, with the increment of the width, the negative added damping enlarges while the inherent fluid damping decreases. Further, the damping ratio of the FSI system decreases accordingly. To summarize, the geometric parameters of the beam have a complicated effect on the damping characteristics of the FSI system which cannot be predicted by the inviscid method, while the two-way FSI simulation in time domain can get satisfactory results in both frequency and damping analysis.

5.3. Time Responses and Time Frequency Analysis. Classically in structural vibration analysis, Fourier transformation is the most widely used technology, but for a signal with variable frequencies, the capability of Fourier transformation is insufficient, as the frequency versus the time cannot be obtained, which is an important characteristic for time-varying systems. Here, time-frequency analysis is carried out to analyze the vibration signals of a time-varying system. In the time-frequency analysis, there are many famous distribution functions, such as short-time Fourier transformation, Hilbert-Huang transformation, and Choi-Williams distribution. Choi-Williams distribution is one of the members of Cohen general time-frequency distributions classes and widely is used with the powerful capability in signal concentration and crossterm suppression [49-52]. Choi-Williams distribution is expressed as follows:

$$
\mathrm{CW}(t, \omega)=\frac{1}{4 \pi^{3 / 2}} \iint \frac{1}{\sqrt{\tau^{2} / \sigma}} s\left(u+\frac{\tau}{2}\right) s^{*}\left(u+\frac{\tau}{2}\right) e^{-\sigma(u-t)^{2} / \tau^{2}-j \tau \omega} d u d \tau,
$$

where $s(u+t / 2)$ denotes the time signal, $s *(u+t / 2)$ represents the complex conjugate function of $s(u+t / 2)$, and $\sigma>0$ is a scale control factor for the smoothness of the transformation. The kernel function has the following form:

$$
\varphi(\theta, \tau)=e^{-\theta^{2} \tau^{2} / \sigma}
$$

The kernel function can improve the crossterm magnitude and time-frequency resolution.

Figures 15(a) and 15(b) demonstrate the density energy spectrum of the responses in Figures 11 and 12, respectively. From Figure 15, it can be found that the frequency increases as the added mass becomes smaller resulting from the descending fluid, and the vibration energy shown in the two figures agrees well with the vibration amplitude in Figures 11 and 12 . In the case of free vibrations, only few signals can be acquired due to the action of the remarkable fluid damping, and the frequency change is not distinguished according to 

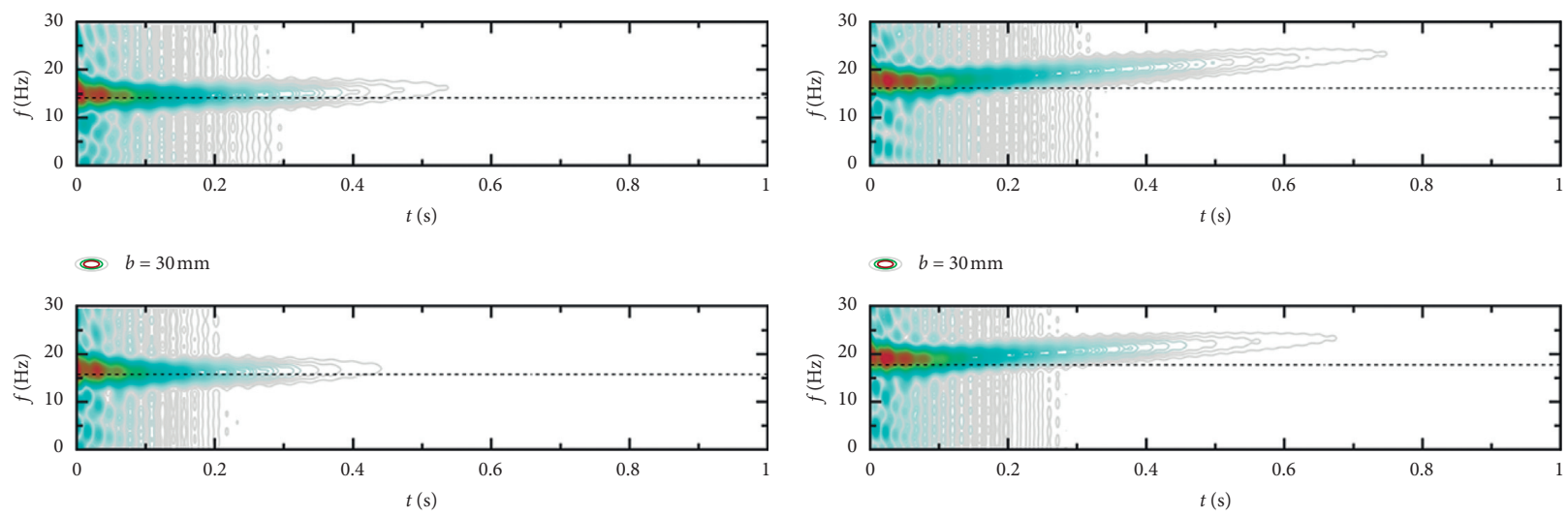

(o) $b=20 \mathrm{~mm}$

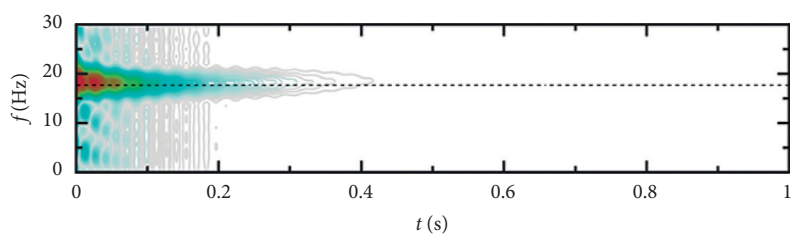

(o) $b=12 \mathrm{~mm}$ (o) $b=20 \mathrm{~mm}$

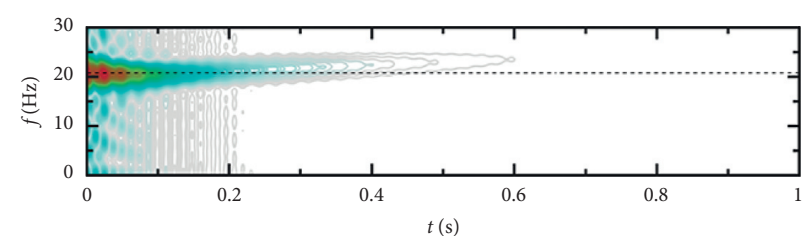

- $b=12 \mathrm{~mm}$

(a)

(b)

Figure 16: Time-frequency analysis of the vibration signals with different widths. (a) Beam I; (b) Beam II.

amplitude frequency curves. However, one can also observe that the vibration phenomenon shows great difference, comparing Figures 15(a) and 15(b). In Figure 15(a), the vibration frequency increases slowly as the descending of the fluid, and the range of the contour lines shrinks as the descending velocity increases, meaning that for Beam I, the vibration energy decreases more quickly with a larger descending velocity of the fluid. For Beam II in Figure 15(b), as the liquid flows out of the container with a larger velocity, the vibration frequency increases rapidly and the vibration energy decreases more slowly. These results confirm that the influences of fluid on the beam are mainly caused by surrounding fluid near the free end.

The density energy spectrums of the response of beam with different widths are displayed in Figures 16(a) and 16(b) for the two types of beams, respectively. For both Beam I and Beam II, when the liquid domain descends the same depth at the same velocity, the vibration frequency increases more for the wider beam. The reason is that the added mass factor increases with the width of the beam, which changes more rapidly with the descending of the liquid.

Comparing Figures 16(a) and 16(b), it can be found that the vibration energy of Beam II decreases more slowly than that of Beam I with the same width, proving that the surrounding fluid mainly affects the beam at the free end again. The contour line exhibits a short range while the submerged beam has a small width, which demonstrates that the damping of the FSI system decreases with the width of the beam. It should be noted that the decrease of the damping is not significant in Figure 16(a) due to the different influences of the width of beam on the inherent damping and added damping.

\section{Conclusions}

In this paper, FSI analysis of cantilever beams vibrating in time-varying fluid domain was mainly focused on. The influence of the time-varying fluid on the vibration of cantilever beams was investigated by experimental measurement and numerical simulations, and the time responses and time-frequency responses were displayed. It is found the fluid acted more on both added mass and damping when the free part of the beam interacts with the fluid, and the following can be concluded from the results:

(1) Besides the influence of the fluid on the vibration frequencies, the time-varying fluid also induced a term which acts like damping, which affected the dynamic responses of the beam significantly. The value of damping-like term is proportional to the moving velocity of the fluid. For the cantilever beam vibrating in a descending fluid with the fixed end submerged, the damping-like term acted as a negative damping, which leaded to instability of vibration of structures; both the time responses and time-frequency responses showed that.

(2) Unlike the beam vibrating in vacuum, the width of the beam has a significant influence on the frequency and damping characteristics of the beam vibrating in time-varying fluid, both the added mass and the added damping increase with the width of the beam, and a viscous method is needed due to the opposite characteristics of the inherent damping of the fluid. In the analysis of the FSI problems with time-varying fluid, the influence of the damping-like term should 
not be neglected. The developed solver is expected to be applicable to simulations of FSI problems interaction with fluid changing irregularly.

\section{Data Availability}

The data used to support the findings of this study are included within the article.

\section{Conflicts of Interest}

The authors declare that they have no conflicts of interest.

\section{Acknowledgments}

This work was supported by the Natural Science Foundation of Shandong Province, China (Grant no. ZR2018LE014), National Natural Science Foundation of China (Grant no. 11702162), and Youth Science and Technology Innovation Program in Higher Education of Shandong Province, China (Grant no. 2019KJB033).

\section{References}

[1] C.-C. Liang, C.-C. Liao, Y.-S. Tai, and W.-H. Lai, "The free vibration analysis of submerged cantilever plates," Ocean Engineering, vol. 28, no. 9, pp. 1225-1245, 2001.

[2] V. H. Vu, M. Thomas, A. A. Lakis et al., Effect of Added Mass on Submerged Vibrated Plates, Canadian Machinery Vibration Association, London, UK, 2007.

[3] Y. Kerboua, A. A. Lakis, M. Thomas, and L. Marcouiller, "Vibration analysis of rectangular plates coupled with fluid," Applied Mathematical Modelling, vol. 32, no. 12, pp. 25702586, 2008.

[4] G. Rezazadeh, M. Fathalilou, R. Shabani, S. Tarverdilou, and S. Talebian, "Dynamic characteristics and forced response of an electrostatically-actuated microbeam subjected to fluid loading," Microsystem Technologies, vol. 15, no. 9, pp. 1355-1363, 2009.

[5] S. Tariverdilo, M. Shahmardani, J. Mirzapour et al., "Asymmetric free vibration of circular plate in contact with incompressible fluid," Applied Mathematical Modelling, vol. 37, no. 1-2, pp. 228-239, 2013.

[6] R. Shabani, H. Hatami, F. G. Golzar, S. Tariverdilo, and G. Rezazadeh, "Coupled vibration of a cantilever micro-beam submerged in a bounded incompressible fluid domain," Acta Mechanica, vol. 224, no. 4, pp. 841-850, 2013.

[7] F. G. Golzar, R. Shabani, H. Hatami, and G. Rezazadeh, "Dynamic response of an electrostatically actuated microbeam in an incompressible viscous fluid cavity," Journal of Microelectromechanical Systems, vol. 23, no. 3, pp. 555-562, 2014.

[8] A. E. Baroudi and F. Razafimahery, "Transverse vibration analysis of Euler-Bernoulli beam carrying point masse submerged in fluid media," International Journal of Engineering \& Technology, vol. 4, no. 2, pp. 369-380, 2015.

[9] C. T. Faria and D. J. Inman, "Modeling energy transport in a cantilevered Euler-Bernoulli beam actively vibrating in Newtonian fluid," Mechanical Systems \& Signal Processing, vol. 45, no. 2, pp. 317-329, 2014.

[10] C. A. V. Eysden and J. E. Sader, "Compressible viscous flows generated by oscillating flexible cylinders," Physics of Fluids, vol. 21, Article ID 013104, 2009.
[11] Ö. Civalek, B. Uzun, M. Ö. Yaylı et al., "Size-dependent transverse and longitudinal vibrations of embedded carbon and silica carbide nanotubes by nonlocal finite element method," The European Physical Journal Plus, vol. 135, Article ID 381, 2020.

[12] F. Ebrahimi, M. R. Barati, and Ö. Civalek, "Application of Chebyshev-Ritz method for static stability and vibration analysis of nonlocal microstructure-dependent nanostructures," Engineering with Computers, vol. 36, no. 3, pp. 953-964, 2020.

[13] M. H. Jalaei and Ö. Civalek, "On dynamic instability of magnetically embedded viscoelastic porous FG nanobeam," International Journal of Engineering Science, vol. 143, pp. 14-32, 2019.

[14] S. Dastjerdi, B. Akgöz, and Ö. Civalek, "On the effect of viscoelasticity on behavior of gyroscopes," International Journal of Engineering Science, vol. 149, Article ID 103236, 2020.

[15] H. Hosaka, K. Itao, and S. Kuroda, "Damping characteristics of beam-shaped micro-oscillators," Sensors and Actuators A Physical, vol. 49, no. 1-2, pp. 87-95, 1995.

[16] S. Kirstein, M. Mertesdorf, and M. Schönhoff, "The influence of a viscous fluid on the vibration dynamics of scanning nearfield optical microscopy fiber probes and atomic force microscopy cantilevers," Journal of Applied Physics, vol. 84, no. 4, pp. 1782-1790, 1998.

[17] J. E. Sader, "Frequency response of cantilever beams immersed in viscous fluids with applications to the atomic force microscope," Journal of Applied Physics, vol. 84, no. 1, pp. 64-76, 1998.

[18] J. W. M. Chon, P. Mulvaney, and J. E. Sader, "Experimental validation of theoretical models for the frequency response of atomic force microscope cantilever beams immersed in fluids," Journal of Applied Physics, vol. 87, no. 8, pp. 3978-3988, 2000.

[19] C. A. V. Eysden and J. E. Sader, "Small amplitude oscillations of a flexible thin blade in a viscous fluid: exact analytical solution," Physics of Fluids, vol. 18, no. 12, Article ID 123102, 2006.

[20] M. Aureli, M. E. Basaran, and M. Porfiri, "Nonlinear finite amplitude vibrations of sharp-edged beams in viscous fluids," Journal of Sound and Vibration, vol. 331, no. 7, pp. 1624-1654, 2012.

[21] M. Aureli, C. Pagano, and M. Porfiri, "Nonlinear finite amplitude torsional vibrations of cantilevers in viscous fluids," Journal of Applied Physics, vol. 111, no. 12, Article ID 124915, 2012.

[22] C. N. Phan, M. Aureli, and M. Porfiri, "Finite amplitude vibrations of cantilevers of rectangular cross sections in viscous fluids," Journal of Fluids and Structures, vol. 40, no. 7 , pp. 52-69, 2013.

[23] C. Intartaglia, L. Soria, and M. Porfiri, "Hydrodynamic coupling of two sharp-edged beams vibrating in a viscous fluid," Proceedings of the Royal Society A Mathematical Physical \& Engineering Sciences, vol. 470, Article ID 20130397, 2014.

[24] M. Jalalisendi, R. Panciroli, Y. Cha et al., "A particle image velocimetry study of the flow physics generated by a thin lamina oscillating in a viscous fluid," Journal of Applied Physics, vol. 115, no. 5, Article ID 054901, 2014.

[25] C. A. V. Eysden and J. E. Sader, "Frequency response of cantilever beams immersed in compressible fluids with applications to the atomic force microscope," Journal of Applied Physics, vol. 106, Article ID 094904, 2009. 
[26] C.-Y. Liao and C.-C. Ma, "Vibration characteristics of rectangular plate in compressible inviscid fluid," Journal of Sound and Vibration, vol. 362, pp. 228-251, 2016.

[27] C. P. Green and J. E. Sader, "Frequency response of cantilever beams immersed in viscous fluids near a solid surface with applications to the atomic force microscope," Journal of Applied Physics, vol. 98, no. 11, Article ID 114913, 2005.

[28] C. P. Green and J. E. Sader, "Small amplitude oscillations of a thin beam immersed in a viscous fluid near a solid surface," Physics of Fluids, vol. 17, no. 7, Article ID 073102, 2005.

[29] T. Naik, E. K. Longmire, and S. C. Mantell, "Dynamic response of a cantilever in liquid near a solid wall," Sensors and Actuators A: Physical, vol. 102, no. 3, pp. 240-254, 2003.

[30] Z. G. Liu, Y. Liu, and J. Lu, "Fluid-structure interaction of single flexible cylinder in axial flow," Computers \& Fluids, vol. 56, no. 15, pp. 143-151, 2012.

[31] A. L. Facci and M. Porfiri, "Analysis of three-dimensional effects in oscillating cantilevers immersed in viscous fluids," Journal of Fluids and Structures, vol. 38, pp. 205-222, 2013.

[32] M. R. Kramer, Z. Liu, and Y. L. Young, "Free vibration of cantilevered composite plates in air and in water," Composite Structures, vol. 95, pp. 254-263, 2013.

[33] A. Ergin and B. Uğurlu, "Linear vibration analysis of cantilever plates partially submerged in fluid," Journal of Fluids and Structures, vol. 17, no. 7, pp. 927-939, 2003.

[34] D. Sedlar, Ž. Lozina, and D. Vučina, "Experimental investigation of the added mass of the cantilever beam partially submerged in water," Tehnicki Vjesnik, vol. 18, pp. 589-594, 2011.

[35] A. Hossain and A. Mian, "Numerical analysis to predict dynamic response of mini cantilever beam submerged in viscous fluids," American Society of Mechanical Engineers (ASME), vol. 18, pp. 1-6, 2011.

[36] A. Hossain, A. Mishty, and A. Mian, "Numerical analysis for design optimization of microcantilever beams for measuring rheological properties of viscous fluid," Finite Elements in Analysis and Design, vol. 68, pp. 1-9, 2013.

[37] M. K. Kwak and D.-H. Yang, "Free vibration analysis of cantilever plate partially submerged into a fluid," Journal of Fluids and Structures, vol. 40, no. 7, pp. 25-41, 2013.

[38] R. Ali, J. Talookolaei, and S. L. Imani, "Free vibration analysis of a delaminated beam-fluid interaction system," Ocean Engineering, vol. 107, no. 1, pp. 186-192, 2015.

[39] G. T. Michaltsos and A. N. Kounadis, "The effects of centripetal and Coriolis forces on the dynamic response of light bridges under moving loads," Journal of Vibration and Control, vol. 7, no. 3, pp. 315-326, 2001.

[40] G. T. Michaltsos, "Dynamic behaviour of a single-span beam subjected to loads moving with variable speeds," Journal of Sound and Vibration, vol. 258, no. 2, pp. 359-372, 2002.

[41] B. Su, L. Zhong, X.-K. Wang et al., "Numerical simulation of patient-specific left ventricular model with both mitral and aortic valves by FSI approach," Computer Methods and Programs in Biomedicine, vol. 113, no. 2, pp. 474-482, 2014.

[42] S. K. Dahl, J. Vierendeels, S. Annerel, L. R. Hellevik, and B. Skallerud, "FSI simulation of asymmetric mitral valve dynamics during diastolic filling," Computer Methods in Biomechanics and Biomedical Engineering, vol. 15, no. 2, pp. 121-130, 2012.

[43] G. Ma, F. Shi, and J. T. Kirby, "Shock-capturing non-hydrostatic model for fully dispersive surface wave processes," Ocean Modelling, vol. 43-44, pp. 22-35, 2012.

[44] F. Gallerano, G. Cannata, and F. Palleschi, "Hydrodynamic effects produced by submerged breakwaters in a coastal area with a curvilinear shoreline," Journal of Marine Science and Engineering, vol. 7, Article ID 337, 2019.

[45] C. W. Hirt and B. D. Nichols, "Volume of fluid (VOF) method for the dynamics of free boundaries," Journal of Computational Physics, vol. 39, no. 1, pp. 201-225, 1981.

[46] M. Derakhti, J. T. Kirby, F. Shi et al., "HWAVE: consistent boundary conditions and turbulence modeling," Ocean Modelling, vol. 106, pp. 121-130, 2016.

[47] W. Aniszewski, T. Ménard, and M. Marek, "Volume of Fluid (VOF) type advection methods in two-phase flow: a comparative study," Computers \& Fluids, vol. 97, no. 6, pp. 52-73, 2014.

[48] D. Youngs, Time-Dependent Multi-Material Flow with Large Fluid Distortion, Academic Press, New York, NY, USA, 1982.

[49] A. F. Hussein, S. J. Hashim, A. F. A. Aziz et al., "Performance evaluation of time-frequency distributions for ECG signal analysis," Journal of Medical Systems, vol. 42, Article ID 15, 2018.

[50] L. Stanković, M. Daković, and T. Thayaparan, Time-frequency Signal Analysis with Applications, Artech House, Boston, MA, USA, 2013.

[51] F. Hlawatsch, T. G. Manickam, R. L. Urbanke, and W. Jones, "Smoothed pseudo-Wigner distribution, Choi-Williams distribution, and cone-kernel representation: ambiguity-domain analysis and experimental comparison," Signal Processing, vol. 43, no. 2, pp. 149-168, 1995.

[52] C. Ma, X. Zhang, Y. Luo, and S. Zhang, "Dynamic behaviors of flexible fluid containers with fuel consumption," Proceedings of the Institution of Mechanical Engineers, Part C: Journal of Mechanical Engineering Science, vol. 229, no. 15, pp. 27372749, 2015. 